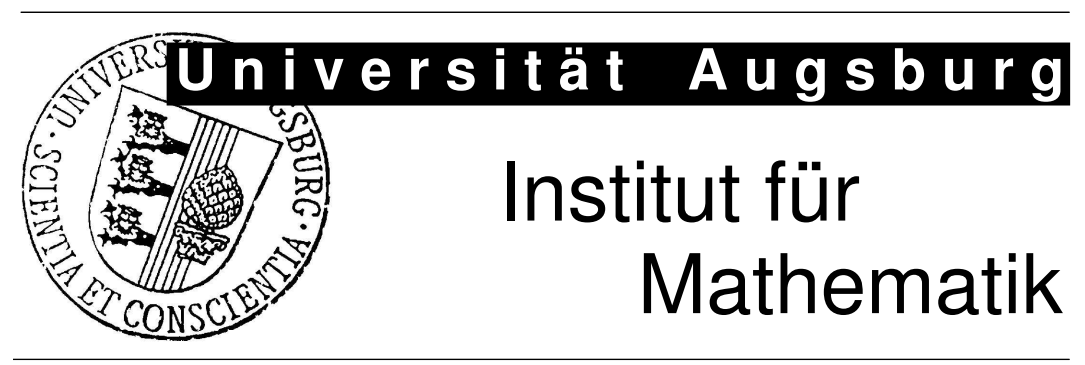

Markus Göhl, Karl Heinz Borgwardt

The Average Number of Pivot Steps of the Simplex-Algorithm Based on a Generalized Rotation-Symmetry-Model 


\section{Impressum:}

\section{Herausgeber:}

Institut für Mathematik

Universität Augsburg

86135 Augsburg

http://www . math. uni-augsburg.de/de/forschung/preprints.html

\section{ViSdP:}

Markus Göhl

Institut für Mathematik

Universität Augsburg

86135 Augsburg

Preprint: Sämtliche Rechte verbleiben den Autoren (C) 2014 


\title{
The Average Number of Pivot Steps of the Simplex-Algorithm Based on a Generalized Rotation-Symmetry-Model
}

\author{
(Preprint)
}

Markus Göhl \& Karl Heinz Borgwardt

\author{
Institut für Mathematik \\ Universität Augsburg
}

2014

\begin{abstract}
This paper deals with the average-case-analysis of the number of pivot steps required by the simplex method. It generalizes results of Borgwardt (who worked under the assumpution of the rotation-symmetry-model) for the shadow-vertex-algorithm to so-called cylindric distributions. Simultaneously it allows to analyze an extended dimension-by-dimension-algorithm, which solves linear programing problems with arbitrary capacity bounds $b$ in the restrictions $A x \leq b$, whereas the model used by Borgwardt required strictly positive right hand sides $b$. These extensions are achieved by solving a problem of stochastic geometry closely related to famous results of Renyi and Sulanke, namely: Assume that $a_{1}, \ldots, a_{m}$ are uniformly distributed in a cylinder. How many facets of $\operatorname{conv}\left(a_{1}, \ldots, a_{m}, 0\right)$ will be intersected by a two-dimensional shadow plane along the axis of the cylinder. The consequence of these investigations is that the upper bounds of Borgwardt (under his model) still apply when we accept distributions with arbitrary right hand sides.
\end{abstract}




\section{Introduction}

Today the simplex-algorithm is still one of the most important algorithms for solving linear programs. It was presented first by George Dantzig [6], [7] in the 1950s and then became very famous and popular. Since a great gap between practical experience and theoretical worst case has been observed, first discovered by Klee and Minty [10], one started to analyze the average runtime of the algorithm. The first pioneering results in this field came from Borgwardt [5], [3], [4], who showed that the expected number of required pivot steps is polynomial in the restriction number $m$ and the dimension $n$ for the shadow-vertex-algorithm, a special variant of the simplex-algorithm. For that purpose he observed canonical problems maximize $v^{T} x$ subject to $A x \leq b$ with a constant right side of the inequalities $b=\mathbb{1},{ }^{1}$ what guarantees the feasibility of the problems. As stochastical model he used the well-known rotation-symmetry-model, which we will define later. The aim of this work is to generalize this result in the way that we will allow arbitrary values of the right sides (so that the problems can also become infeasible). We will concentrate on the asymptotic case, i. e. we assume the dimension $n$ as constant and let the restriction number $m$ tend to infinity. For the purpose of sharper calculations we will focus on a special rotational symmetric distribution family, which is representative for general rotational symmetric distributions and for which sharp bounds were already proven by Höfner [9]. For a comprehensive historical overview about the average-case-analysis of the simplex-algorithm we refer to [4]. The contents of this paper are essentially based on the dissertation of Markus Göhl [8], supplemented by some improvements of partial results. Besides we give a much easier proof of one central Lemma. Newly, in addition to the calculated upper bound we are able to state an asymptotic lower bound for the analyzed expectation value.

\subsection{The problem and its motivation}

Before we start let us agree on the following notation for vectors: We denote a series of different vectors by $a_{1}, \ldots, a_{m}$, while we write $a^{1}, \ldots, a^{n}$ for the components of one such vector $a$. So we have to write $\left(a^{3}\right)^{2}$ for the squared value of the third component of the vector $a$, but in this context there will be almost no exponents, so an upper index cannot be misunderstood. Now, let us start with the problem we want to analyze in this paper. For linear programs of the form

$$
\begin{array}{ll}
\max & v^{T} x \\
\text { s. t. } & a_{1}^{T} x \leq 1, \ldots, a_{m}^{T} x \leq 1 \\
\text { with } & a_{1}, \ldots, a_{m}, v, x \in \mathbb{R}^{n} \text { and } m \geq n
\end{array}
$$

the average runtime for solving such problems with the shadow-vertex-algorithm, a special variant of the simplex-algorithm, has been elaborately outlined by Borgwardt

\footnotetext{
${ }^{1}$ Here, $\mathbb{1}$ is the vector containing 1 in each component.
} 
[4] using the rotation-symmetry-model.

$a_{1}, \ldots, a_{m}, v \in \mathbb{R}^{n} \backslash\{0\}$ are distributed independently, identically and rotationally symmetric.

In this model we may assume the following condition of nondegeneracy of the observed problems, as degenerated problems only occur with probability 0 .

Each subset of $n$ elements out of $\left\{a_{1}, \ldots, a_{m}, v\right\}$ is linearly independent and each subset of $n+1$ elements out of $\left\{a_{1}, \ldots, a_{m}\right\}$ is in general position.

(CoND)

The crucial result for the expected runtime of the shadow-vertex-algorithm under the rotation-symmetry-model by Borgwardt [1] is the following.

Theorem 1.1. Let $a_{1}, \ldots, a_{m}, v \in \mathbb{R}^{n} \backslash\{0\}$ be generated according to the rotationsymmetry-model. Then

$$
\mathbb{E}_{m, n}[S] \leq C \cdot m^{\frac{1}{n-1}} \cdot n^{2}
$$

where $C$ is a constant depending not on $m$ and $n$.

Here, $S$ is the number of shadow vertices and therefore a simple upper bound for the required number of pivot steps of the shadow-vertex-algorithm for proceeding from one given start vertex to the optimal vertex (phase II). ${ }^{2}$ One essential feature of problems of type $\left(L P_{1}\right)$ is the positive bound on the right side of the constraints, which guarantees the feasibility of these problems. As this nice feature is a limitation of generality, of course, we want to observe general linear problems of the form

$$
\begin{array}{ll}
\max & v^{T} x \\
\text { s. t. } & a_{1}^{T} x \leq 1-\beta^{1}, \ldots, a_{m}^{T} x \leq 1-\beta^{m} \\
\text { with } & a_{1}, \ldots, a_{m}, v, x \in \mathbb{R}^{n}, \beta^{1}, \ldots, \beta^{m} \in \mathbb{R} \text { and } m \geq n+1
\end{array}
$$

in this paper. So from now on, we cannot be sure that there is a feasible solution for the problem at all. We denote the feasible region of such a problem by $Z$ and remark that the problem class $\left(L P_{b}\right)$ contains the special class $\left(L P_{1}\right)$ what can be seen by setting $\beta^{i}=0$ for all $i=1, \ldots, m$. We now want to use the knowledge about programs of the type $\left(L P_{1}\right)$ in order to solve programs of the type $\left(L P_{b}\right)$. For the solution of $\left(L P_{1}\right)$ we apply the dimension-by-dimension-algorithm of Borgwardt as it is described in [4]. There are generally two possible cases:

1. We get an optimal vertex $\bar{x}$ (primal view) respectively a facet $\operatorname{conv}\left(a_{\Delta^{1}}, \ldots, a_{\Delta^{n}}\right)$ of the dual polyhedron $Y:=\operatorname{conv}\left(0, a_{1}, \ldots, a_{m}\right)$ which is intersected by $\mathbb{R}^{+} v$ (dual view).

\footnotetext{
${ }^{2}$ More details about the quantity $S$ can be read in [4].
} 
2. $v^{T} x$ is unbounded on the primal polyhedron

$$
X:=\left\{x \in \mathbb{R}^{n} \mid a_{1}^{T} x \leq 1, \ldots, a_{m}^{T} x \leq 1\right\}
$$

(primal view) respectively the ray $\mathbb{R}^{+} v$ does not cut $Y$ (dual view).

Here, $\Delta=\left\{\Delta^{1}, \ldots, \Delta^{n}\right\} \subseteq\{1, \ldots, m\}$ is a collection of indices of vectors $a_{i}$ such that $a_{\Delta^{1}}, \ldots, a_{\Delta^{n}}$ define a basis of $\mathbb{R}^{n}$. In the second case, there is an increasing free direction, i. e.

$$
\exists d \in \mathbb{R}^{n} \text { with } a_{1}^{T} d \leq 0, \ldots, a_{m}^{T} d \leq 0 \text { and } v^{T} d>0 .
$$

This $d$ also is an increasing free direction for $\left(L P_{b}\right)$. So $\left(L P_{b}\right)$ is either infeasible or (feasible and) unbounded, so there definitely is no optimal solution what allows us to interrupt the algorithm in this case. Much more interesting is the first case, when there is an optimal vertex of $X$ respectively an optimal facet of $Y$. Of course, we can rewrite our inequalities

$$
a_{1}^{T} x \leq 1-\beta^{1}, \ldots, a_{m}^{T} x \leq 1-\beta^{m}
$$

to

$$
a_{1}^{T} x+\beta^{1} \leq 1, \ldots, a_{m}^{T} x+\beta^{m} \leq 1 .
$$

Now, we augment the vector of variables $x \in \mathbb{R}^{n}$ with one additional component and obtain for $\tilde{x}=\left(x^{T}, \tilde{x}^{n+1}\right)^{T} \in \mathbb{R}^{n+1}$ the new problem

$\max \quad \tilde{v}^{T} \tilde{x}=v^{T} x+0 \cdot \tilde{x}^{n+1}$

s. t. $\quad \tilde{a}_{1}^{T} \tilde{x}=a_{1}^{T} x+\beta^{1} \tilde{x}^{n+1} \leq 1, \ldots, \tilde{a}_{m}^{T} \tilde{x}=a_{m}^{T} x+\beta^{m} \tilde{x}^{n+1} \leq 1$

with $\quad \tilde{a}_{1}, \ldots, \tilde{a}_{m}, \tilde{v}, \tilde{x} \in \mathbb{R}^{n+1}$ and $m \geq n+1$.

Writing $\tilde{X}$ for the feasible region of $\left(L P_{a}\right)$ and $\tilde{\Pi}_{k}$ for the canonical projection from $\mathbb{R}^{n+1}$ onto $\mathbb{R}^{k}$, precised by

$$
\tilde{\Pi}_{k}: \mathbb{R}^{n+1} \rightarrow \mathbb{R}^{k}, \quad\left(x^{1}, \ldots, x^{n+1}\right) \mapsto\left(x^{1}, \ldots, x^{k}\right),
$$

it follows

$$
Z=\tilde{\Pi}_{n}\left(\tilde{X} \cap\left\{\tilde{x} \in \mathbb{R}^{n+1} \mid \tilde{x}^{n+1}=1\right\}\right)
$$

and

$$
X=\tilde{\Pi}_{n}\left(\tilde{X} \cap\left\{\tilde{x} \in \mathbb{R}^{n+1} \mid \tilde{x}^{n+1}=0\right\}\right) .
$$

The problem $\left(L P_{a}\right)$ is of the type $\left(L P_{1}\right)$ again, so we can solve it. Besides $Z$ is empty, if and only if

$$
\tilde{X} \cap\left\{\tilde{x} \in \mathbb{R}^{n+1} \mid \tilde{x}^{n+1}=1\right\}
$$

is empty. Therefore, the decision whether $Z$ is empty becomes an optimization problem, namely the maximization of $e_{n+1}^{T} \tilde{x}=\tilde{x}^{n+1}$ on $\tilde{X}$. Since we have

$$
Z=\emptyset \Longleftrightarrow \max \left\{e_{n+1}^{T} \tilde{x} \mid \tilde{x} \in \tilde{X}\right\}<1 \text {. }
$$


As we already have solved $\left(L P_{1}\right)$, we know the $v$-optimal vertex $x_{v}$ of $X$. Its embedding $\tilde{x}_{v}=\left(x_{v}^{T}, 0\right)^{T}$ lies on the one hand on an edge of $\tilde{X}$ and it is on the other hand optimal with respect to $\tilde{v}^{T} \tilde{x}$ on $\tilde{X} \cap\left\{\tilde{x} \in \mathbb{R}^{n+1} \mid e_{n+1}^{T} \tilde{x}=e_{n+1}^{T} \tilde{x}_{v}=0\right\}$. So $\tilde{x}_{v}$ lies on a shadow-vertex-path relative to $\tilde{v}^{T} \tilde{x}$ and $e_{n+1}^{T} \tilde{x}$. Thus, we only have to find a vertex on the edge which contains $\tilde{x}_{v}$ and then start the shadow-vertex-algorithm using the shadow plane $\operatorname{span}\left(\tilde{v}, e_{n+1}\right)$. If the optimal value is less than $1,\left(L P_{b}\right)$ is infeasible. Otherwise we can calculate a solution $\tilde{x}_{1}$ with $e_{n+1}^{T} \tilde{x}_{1}=1$ and get the optimal solution $\tilde{\Pi}_{n}\left(\tilde{x}_{1}\right)$ of $\left(L P_{b}\right)$, as $\tilde{x}_{1}$ is optimal with respect to $\tilde{v}^{T} \tilde{x}$ on $\tilde{X} \cap\left\{\tilde{x} \in \mathbb{R}^{n+1} \mid e_{n+1}^{T} \tilde{x}=1\right\}$. Finally, we formulate the augmented dimension-by-dimension-algorithm on the basis of the original algorithm of Borgwardt [4]. There we will use the intuitive notation $\Pi_{k}: \mathbb{R}^{n} \rightarrow \mathbb{R}^{k}, \quad\left(x^{1}, \ldots, x^{n}\right) \mapsto\left(x^{1}, \ldots, x^{k}\right)$ and $\tilde{Y}:=\operatorname{conv}\left(0, \tilde{a}_{1}, \ldots, \tilde{a}_{m}\right)$.

Algorithm 1.2 (Augmented dimension-by-dimension-algorithm).

1. Find a facet $\Pi_{2}\left(\operatorname{conv}\left(a_{\Delta^{1}}, a_{\Delta^{2}}\right)\right)$ of $\Pi_{2}(Y)$.

2. Find the facet of $\Pi_{2}(Y)$ which is intersected by the ray $\mathbb{R}^{+} \Pi_{2}(v)$. If there is no such facet, go to $\%$.

3. If $k=n$, go to 8 , otherwise set $k=k+1$.

4. The optimal facet $\Pi_{k-1}\left(\operatorname{conv}\left(a_{\Delta^{1}}, \ldots, a_{\Delta^{k-1}}\right)\right)$ of $L P_{k-1}$ is available. Find a vector $a_{i}$ with $i \notin\left\{\Delta^{1}, \ldots, \Delta^{k-1}\right\}$ so that $\Pi_{k}\left(\operatorname{conv}\left(a_{\Delta^{1}}, \ldots, a_{\Delta^{k-1}}, a_{i}\right)\right)$ is a facet of $\Pi_{k}(Y)$.

5. Start the shadow-vertex-algorithm at $\Pi_{k}\left(\operatorname{conv}\left(a_{\Delta^{1}}, \ldots, a_{\Delta^{k-1}}, a_{i}\right)\right)$ and find the optimal facet $\Pi_{k}\left(\operatorname{conv}\left(a_{\Delta^{1}}, \ldots, a_{\Delta^{k}}\right)\right)$, which is intersected by the ray $\mathbb{R}^{+} \Pi_{k}(v)$. Use $\operatorname{span}\left(\Pi_{k}\left(e_{k}\right), \Pi_{k}(v)\right)$ as shadow plane. If there is no such facet, go to $\%$.

6. Go to 3.

7. The whole problem has no solution. Print "Problem has no optimal vertex" and go to 9 .

8. The optimal facet of $L P_{n}$ is available. Print the corresponding optimal vertex $x$ and the optimal value $v^{T} x$. Go to 10.

9. STOP.

10. The optimal facet $\tilde{\Pi}_{n}\left(\operatorname{conv}\left(\tilde{a}_{\Delta^{1}}, \ldots, \tilde{a}_{\Delta^{n}}\right)\right)$ of $\tilde{\Pi}_{n}(\tilde{Y})$ is available. Find a vector $\tilde{a}_{i}$ with $i \notin\left\{\Delta^{1}, \ldots, \Delta^{n}\right\}$ so that $\operatorname{conv}\left(\tilde{a}_{\Delta^{1}}, \ldots, \tilde{a}_{\Delta^{n}}, \tilde{a}_{i}\right)$ is a facet of $\tilde{Y}$.

11. Start the shadow-vertex-algorithm at $\operatorname{conv}\left(\tilde{a}_{\Delta^{1}}, \ldots, \tilde{a}_{\Delta^{n}}, \tilde{a}_{i}\right)$ and maximize $e_{n+1}^{T} \tilde{x}$. Use $\operatorname{span}\left(\tilde{v}, e_{n+1}\right)$ as shadow plane. Once $e_{n+1}^{T} \tilde{x} \geq 1$, print $\tilde{a}_{\Delta^{1}}, \ldots, \tilde{a}_{\Delta^{n+1}}$. Delete the $\tilde{a}_{\Delta^{i}}$ which entered the basis last and print $\tilde{a}_{\Delta^{1}}, \ldots, \tilde{a}_{\Delta^{i-1}}, \tilde{a}_{\Delta^{i+1}}, \ldots, \tilde{a}_{\Delta^{n+1}}$. 
12. Calculate the solution $z$ of the system of linear equations

$$
\begin{gathered}
a_{\Delta^{1}}^{T} x=1-\beta^{\Delta^{1}}, \ldots, a_{\Delta^{i-1}}^{T} x=1-\beta^{\Delta^{i-1}}, \\
a_{\Delta^{i+1}}^{T} x=1-\beta^{\Delta^{i+1}}, \ldots, a_{\Delta^{n+1}}^{T} x=1-\beta^{\Delta^{n+1}}
\end{gathered}
$$

and print $z$. This $z \in Z$ is a solution of $\left(L P_{b}\right)$. Go to 9 .

\subsection{An integral formula for the desired expectation value}

We are now interested in the expected number of required pivot steps of the complete algorithm. We denote that number by $s_{t}$. For the original dimension-by-dimensionalgorithm the following result of Borgwardt [1] is already known.

Theorem 1.3. Let $a_{1}, \ldots, a_{m}, v \in \mathbb{R}^{n}$ be generated according to the rotation-symmetrymodel. Then for the expected number of shadow vertices in stage $k$ (denoted by $S^{(k)}$ ) of the algorithm we know that

$$
\mathbb{E}_{m, n}\left[S^{(k)}\right] \leq C_{1} \cdot m^{\frac{1}{n-1}} \cdot n \cdot k .
$$

Furthermore, for the number of necessary pivot steps of the whole algorithm $s_{t}$ we know

$$
\mathbb{E}_{m, n}\left[s_{t}\right] \leq C_{2} \cdot m^{\frac{1}{n-1}} \cdot n^{3} .
$$

Here, $C_{1}$ and $C_{2}$ are constants depending not on $m$ and $n$.

The second part of this result uses the fact that there are $n$ stages in the algorithm, so the result for one stage is multiplied with $n$ what is a rough estimation for the whole effort. Actually, Höfner [9] could show that the effort for the complete algorithm only has an order of growth like $m^{\frac{1}{n-1}} \cdot n^{\frac{5}{2}}$ instead of $m^{\frac{1}{n-1}} \cdot n^{3}$ for the asymptotic case, i. e. $m$ tends to infinity, while the dimension $n$ is constant. This is due to the fact that the effort for the later stages is much lower than for the earlier stages, since the path from the $k$-dimensional optimal vertex to the $(k+1)$-dimensional optimal vertex is relatively short, as only one additional dimension is observed. The effect of that relative efficiency increases with the observed dimension $k$. So in total, the factor $\sqrt{n}$ can be saved by this effect. As we have augmented the dimension-by-dimension-algorithm by one additional stage in essence, the result above still holds, if

$$
\tilde{a}_{1}=\left(\begin{array}{c}
a_{1} \\
\beta^{1}
\end{array}\right), \ldots, \tilde{a}_{m}=\left(\begin{array}{c}
a_{m} \\
\beta^{m}
\end{array}\right), \tilde{v}=\left(\begin{array}{c}
v \\
\beta^{0}
\end{array}\right) \in \mathbb{R}^{n+1}
$$

are generated according to the rotation-symmetry-model. But that does not seem to be reasonable because it would generate a stochastical dependence between $a_{i}$ and $\beta^{i}$. There is only one special case for which we can use the known result without difficulty. 
Theorem 1.4. If $a_{1}, \ldots, a_{m}, v \in \mathbb{R}^{n}$ and $\beta^{1}, \ldots, \beta^{m} \in \mathbb{R}$ are distributed independently, identically, normally, then holds

$$
\mathbb{E}_{m, n}\left[s_{t}\right] \leq C \cdot m^{\frac{1}{n}} \cdot(n+1)^{3}
$$

where $C$ is a constant depending not on $m$ and $n$.

Proof. If $a_{1}, \ldots, a_{m}, v \in \mathbb{R}^{n}$ and $\beta^{1}, \ldots, \beta^{m} \in \mathbb{R}$ are distributed independently, identically, normally, then $\tilde{a}_{1}, \ldots, \tilde{a}_{m}, \tilde{v}$ are generated according to the rotation-symmetrymodel so that Theorem 1.3 is applicable.

For the general case we define the augmented rotation-symmetry-model (RSMA) as follows.

$$
\begin{aligned}
& a_{1}, \ldots, a_{m}, v \in \mathbb{R}^{n} \backslash\{0\}, \beta^{1}, \ldots, \beta^{m} \in \mathbb{R} \text { are stochastically inde- } \\
& \text { pendent. Furthermore, } a_{1}, \ldots, a_{m}, v \text { are distributed identically and } \\
& \text { rotationally symmetric and } \beta^{1}, \ldots, \beta^{m} \text { are distributed uniformly on } \\
& {[-1,1] .}
\end{aligned}
$$

So from now on, our main task is to investigate how many pivot steps are necessary on the average for the last stage of our algorithm, i. e. how many shadow vertices can be expected, when the vectors $\tilde{a}_{1}, \ldots, \tilde{a}_{m}, \tilde{v}$ are generated according to our augmented rotation-symmetry-model. In other words, we are forced to develop an adapted fundamental Theorem for the augmented rotation-symmetry-model corresponding to the crucial Theorem 1.1 of Borgwardt for the classical rotation-symmetry-model.

If $a_{1}, \ldots, a_{m}$ are distributed rotationally symmetric in a ball in $\mathbb{R}^{n}$, then the augmented vectors $\tilde{a}_{1}, \ldots, \tilde{a}_{m}$ are distributed in a cylinder in $\mathbb{R}^{n+1}$. So from a geometrical point of view, it is justified to call such distributions cylinder distributions. In order to avoid misunderstandings we want to clarify some linguistic terms at this point. Based on a unit cylinder

$$
\left\{\left(\begin{array}{l}
x \\
\xi
\end{array}\right) \in \mathbb{R}^{n} \times \mathbb{R} \mid\|x\| \leq 1, \xi \in[-1,1]\right\}
$$

we say cover for the subset of the cylinder with $\xi=1$, bottom for the subset with $\xi=-1$ and barrel for the surface of the cylinder without cover and bottom, i. e. $\|x\|=1$ and $\xi \in(-1,1)$.

For the following considerations we assume that $\beta^{1}, \ldots, \beta^{m} \in \mathbb{R}$ as well as $a_{1}, \ldots, a_{m}, v \in$ $\mathbb{R}^{n} \backslash\{0\}$ (given by a common density function $f$ with compact support) are generated according to (RSMA) and observe the dual polyhedron $\tilde{Y}=\operatorname{conv}\left(0, \tilde{a}_{1}, \ldots, \tilde{a}_{m}\right)$ with

$$
\tilde{a}_{1}=\left(\begin{array}{l}
a_{1} \\
\beta^{1}
\end{array}\right), \ldots, \tilde{a}_{m}=\left(\begin{array}{c}
a_{m} \\
\beta^{m}
\end{array}\right), \tilde{v}=\left(\begin{array}{l}
v \\
0
\end{array}\right) \in \mathbb{R}^{n+1} .
$$


Apart from that we denote the density function of $\tilde{a}_{i} \in \mathbb{R}^{n+1}$ by $\tilde{f}$ so that

$$
\tilde{f}\left(\tilde{a}_{i}\right)=f\left(a_{i}\right) \cdot \hat{f}\left(\beta^{i}\right)
$$

holds, where

$$
\hat{f}: \mathbb{R} \rightarrow \mathbb{R}, \quad \xi \mapsto \begin{cases}\frac{1}{2} & \text { for }-1 \leq \xi \leq 1 \\ 0 & \text { else }\end{cases}
$$

is the density function of the last coordinate. Generally, we assume $m \geq n+1 \geq 4$. The essential quantity we are interested in is the number of facets of the dual polyhedron $\tilde{Y}$ which are intersected by the half-plane $\mathbb{R}^{+} \tilde{v}+\mathbb{R} e_{n+1}$ and whose number we denote by $\tilde{S}$. In the following we take advantage of the fact that in our model a facet is intersected by the half-plane, if and only if exactly two of its boundary $(n-1)$-dimensional simplices are intersected. ${ }^{3}$ Analogously to the approach in [4] we obtain

$$
\begin{aligned}
& \mathbb{E}_{m, n}[\tilde{S}]=\text { "Number of candidates" . } \\
& \text {. "Probability that a candidate is a shadow vertex" } \\
& =\left(\begin{array}{c}
m \\
n+1
\end{array}\right) \cdot P\left(\operatorname{conv}\left(\tilde{a}_{1}, \ldots, \tilde{a}_{n+1}\right) \text { is a facet and } \operatorname{cone}\left(a_{1}, \ldots, a_{n+1}\right) \cap \mathbb{R}^{+} v \neq \emptyset\right) \\
& =\left(\begin{array}{c}
m \\
n+1
\end{array}\right) \cdot \frac{n+1}{2} \cdot \int_{\mathbb{R}^{n}} \int_{\mathbb{R}^{n+1}} \ldots \int_{\mathbb{R}^{n+1}} P\left(\operatorname{conv}\left(\tilde{a}_{1}, \ldots, \tilde{a}_{n+1}\right) \text { is a facet }\right) \cdot \\
& \cdot P\left(\operatorname{cone}\left(a_{1}, \ldots, a_{n}\right) \cap \mathbb{R}^{+} v \neq \emptyset\right) \cdot \tilde{f}\left(\tilde{a}_{1}\right) \ldots \tilde{f}\left(\tilde{a}_{m}\right) f(v) \cdot d \tilde{a}_{1} \ldots d \tilde{a}_{m} d v \\
& =\left(\begin{array}{c}
m \\
n+1
\end{array}\right) \cdot \frac{n+1}{2} \cdot \int_{\mathbb{R}^{n+1}} \ldots \int_{\mathbb{R}^{n+1}} G\left(\tilde{a}_{1}, \ldots, \tilde{a}_{n+1}\right)^{m-(n+1)} . \\
& \cdot W\left(a_{1}, \ldots, a_{n}\right) \cdot \tilde{f}\left(\tilde{a}_{1}\right) \ldots \tilde{f}\left(\tilde{a}_{n+1}\right) \cdot d \tilde{a}_{1} \ldots d \tilde{a}_{n+1}
\end{aligned}
$$

where

$$
\begin{aligned}
G\left(\tilde{a}_{1}, \ldots, \tilde{a}_{n+1}\right) & :=\int_{\mathbb{R}^{n+1}} I_{H^{-}\left(\tilde{a}_{1}, \ldots, \tilde{a}_{n+1}\right)}(\tilde{a}) \tilde{f}(\tilde{a}) d \tilde{a}, \\
W\left(a_{1}, \ldots, a_{n}\right) & :=\frac{\lambda_{n}\left(\operatorname{cone}\left(a_{1}, \ldots, a_{n}\right) \cap \Omega_{n}\right)}{\lambda_{n}\left(\Omega_{n}\right)} .
\end{aligned}
$$

Here we have used that for fixed vectors $\tilde{a}_{1}, \ldots, \tilde{a}_{n+1}$ the events

$$
" \operatorname{conv}\left(\tilde{a}_{1}, \ldots, \tilde{a}_{n+1}\right) \text { is a facet" and " } \operatorname{cone}\left(a_{1}, \ldots, a_{n+1}\right) \cap \mathbb{R}^{+} v \neq \emptyset "
$$

are stochastically independent. The notation corresponds to [4]. So $H^{-}\left(\tilde{a}_{1}, \ldots, \tilde{a}_{n+1}\right)$ describes the half-space which is generated by $\operatorname{conv}\left(\tilde{a}_{1}, \ldots, \tilde{a}_{n+1}\right)$ and contains the origin, by $\lambda_{n}(\cdot)$ we denote the $n$-dimensional Lebesgue-measure and $\Omega_{n}$ is the whole unit

\footnotetext{
${ }^{3}$ We should mention that this claim does not hold for the start-simplex and the optimal-simplex in the simplex path because for these two simplices only one boundary $(n-1)$-dimensional simplex is intersected. But this fact is not essential for our purpose.
} 
ball in $\mathbb{R}^{n}$. Later we will additionally use $\omega_{n}$ for the unit sphere in $\mathbb{R}^{n}$ (surface of the unit ball).

Next we introduce some basic notation for the analysis. Let $H=H\left(\tilde{a}_{1}, \ldots, \tilde{a}_{n+1}\right)$ be the hyperplane which contains $\operatorname{conv}\left(\tilde{a}_{1}, \ldots, \tilde{a}_{n+1}\right)$. Besides $h=h\left(\tilde{a}_{1}, \ldots, \tilde{a}_{n+1}\right)$ describes the distance of $H\left(\tilde{a}_{1}, \ldots, \tilde{a}_{n+1}\right)$ to the origin and $\varphi=\varphi\left(\tilde{a}_{1}, \ldots, \tilde{a}_{n+1}\right)$ the (smaller) angle between the normal vector $\tilde{d}=\tilde{d}\left(\tilde{a}_{1}, \ldots, \tilde{a}_{n+1}\right)$ of $H\left(\tilde{a}_{1}, \ldots, \tilde{a}_{n+1}\right)$ and $e_{n+1}$. And let $\tilde{h}=\tilde{h}\left(\tilde{a}_{1}, \ldots, \tilde{a}_{n+1}\right)$ be the distance from the intersection point of $H\left(\tilde{a}_{1}, \ldots, \tilde{a}_{n+1}\right)$ with the $x^{n+1}$-axis ${ }^{4}$ to the origin. Finally, we write $z$ for the distance of the intersection of the hyperplane $H$ with the cylinder cover to the barrel and $s$ for the distance of the intersection of the hyperplane $H$ with the cylinder barrel to the cover respectively.

We have to distinguish between three constellations on how the hyperplane may intersect the cylinder. We say that the hyperplane belongs to category A, if it intersects the cover and not the bottom of the cylinder (or vice versa), to category B, if it separates the whole cover from the rest of the cylinder, and to category $\mathrm{C}$, if it intersects both cover and bottom of the cylinder. The following table shows, for which sets of the parameters $h$ and $\varphi$ these different cases occur.

\begin{tabular}{c|c|c} 
& $h<|\sin (\varphi)-\cos (\varphi)|$ & $h>|\sin (\varphi)-\cos (\varphi)|$ \\
\hline $0<\varphi<\frac{\pi}{4}$ & category B & category A \\
\hline$\frac{\pi}{4}<\varphi<\frac{\pi}{2}$ & category C & category A
\end{tabular}

For the function $G$, which really only depends on $h$ and $\varphi$, we know

$$
G(h, \varphi)=\int_{-\infty}^{\infty} \ldots \int_{-\infty}^{\infty} \int_{-\infty}^{\frac{h-x^{1} \cdot \sin (\varphi)}{\cos (\varphi)}} f(x) \cdot \hat{f}\left(\tilde{x}^{n+1}\right) \cdot d \tilde{x}^{n+1} d \tilde{x}^{n} \ldots d \tilde{x}^{1}
$$

what is described in detail in [8]. Performing a transformation of coordinates such that the normal vector of the hyperplane has the form $\tilde{d}=\left(\delta^{1}, 0, \ldots, 0, \delta^{n+1}\right)^{T}$ yields $^{5}$

$$
\begin{aligned}
\mathbb{E}_{m, n}[\tilde{S}]=\left(\begin{array}{c}
m \\
n+1
\end{array}\right) & \cdot(n+1) \cdot \lambda_{n-1}\left(\omega_{n}\right) . \\
& \cdot \int_{0}^{\frac{\pi}{2}} \int_{0}^{\sin (\varphi)+\cos (\varphi)} G(h, \varphi)^{m-(n+1)} \cdot \frac{(\sin \varphi)^{n-1}}{(\cos \varphi)^{n+2}} \cdot \Lambda(h, \varphi) \cdot d h d \varphi
\end{aligned}
$$

\footnotetext{
4 This intersection point exists in our model with probability 1.

5 See [8] for details.
} 


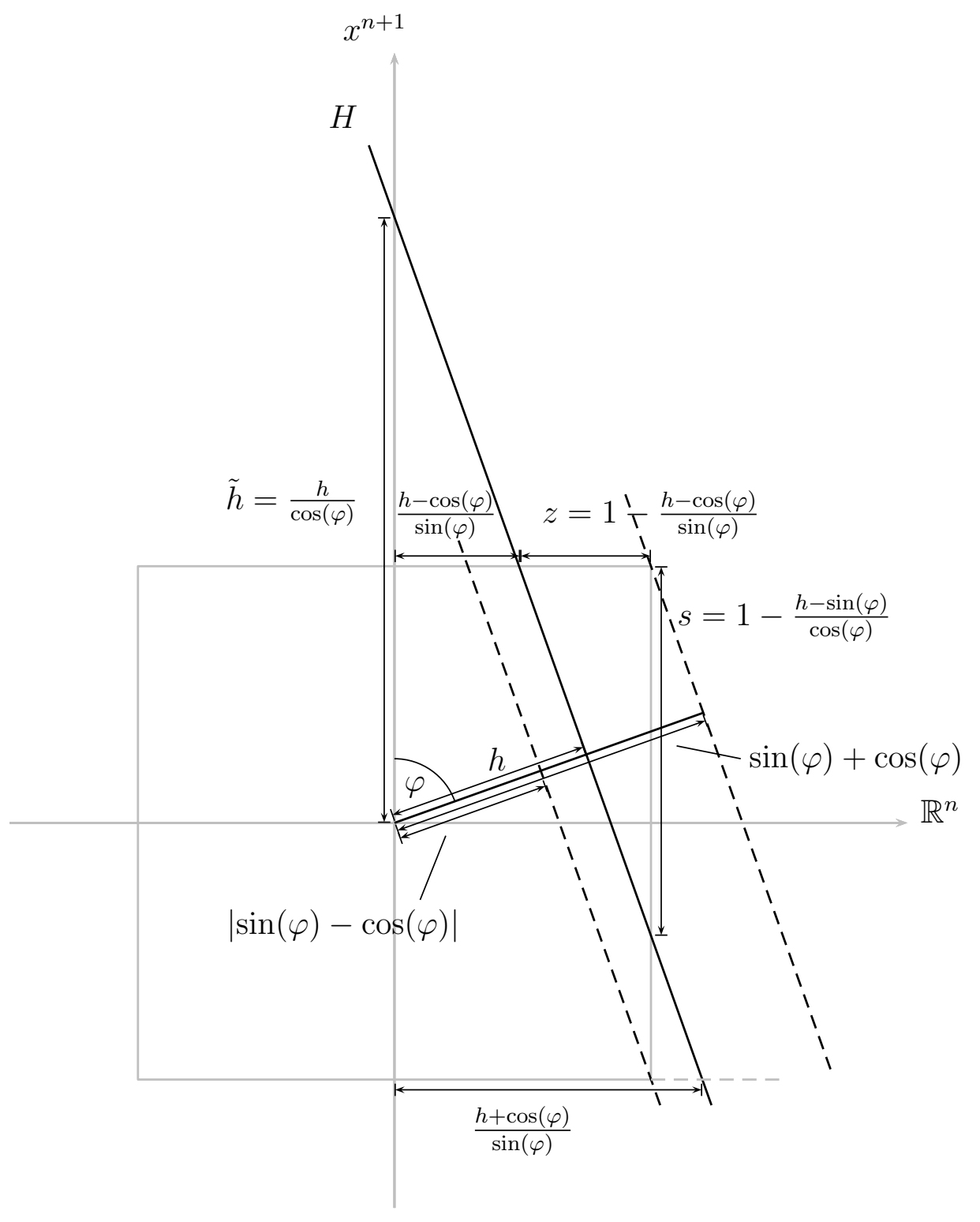

Figure 1.1: Illustration of important quantities, when the hyperplane $H$ intersects the cylinder. 


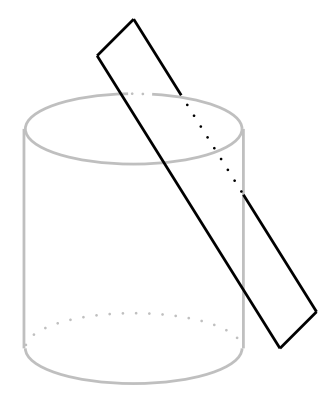

category A

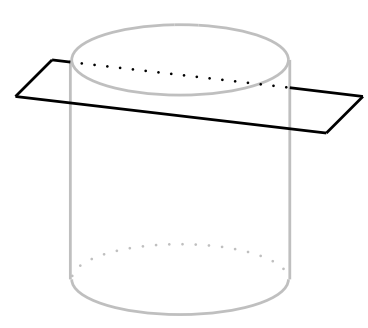

category B

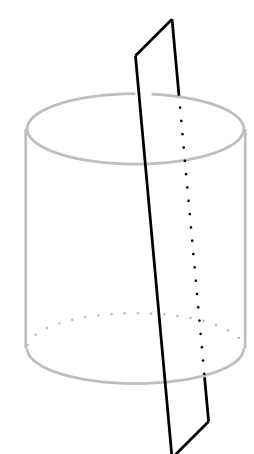

category C

Figure 1.2: Illustration of the different intersection possibilities of the hyperplane with the cylinder.

where

$$
\begin{gathered}
\Lambda(h, \varphi):=\int_{\mathbb{R}^{n}} \ldots \int_{\mathbb{R}^{n}}|\operatorname{det} B| \cdot W\left(b_{1}, \ldots, b_{n}\right) \cdot \tilde{f}\left(\tilde{b}_{1}\right) \ldots \tilde{f}\left(\tilde{b}_{n+1}\right) \cdot d b_{1} \ldots d b_{n+1} \\
=\int_{\mathbb{R}^{n}} \ldots \int_{\mathbb{R}^{n}}|\operatorname{det} B| \cdot W\left(b_{1}, \ldots, b_{n}\right) \cdot f\left(b_{1}\right) \ldots f\left(b_{n+1}\right) \cdot \\
\quad \cdot \hat{f}\left(\frac{h-b_{1}^{1} \sin (\varphi)}{\cos (\varphi)}\right) \ldots \hat{f}\left(\frac{h-b_{n+1}^{1} \sin (\varphi)}{\cos (\varphi)}\right) \cdot d b_{1} \ldots d b_{n+1}
\end{gathered}
$$

and

$$
B:=\left(\begin{array}{cccc}
b_{1}^{1} & \ldots & b_{1}^{n} & 1 \\
\vdots & & \vdots & \vdots \\
b_{n+1}^{1} & \ldots & b_{n+1}^{n} & 1
\end{array}\right)
$$

\section{Asymptotic analysis of the number of shadow vertices in the cylinder and of the last stage of the augmented dimension-by-dimension-algorithm}

In this section we want to analyze the integral formula of the previous section for the asymptotic case, that means that the dimension $n$ is assumed to be constant, while the 
restriction number $m$ tends to infinity. For this purpose we will partition the integral into different pieces which will all be analyzed on their own. In order to get preferably sharp upper bounds for all integrals we will analyze especially one particular family of distributions, which allows us to perform very accurate calculations on multiple integrals. This subclass is representative for all the variety in the set of rotationally symmetric distributions. The members of that family are defined and specified by their radial distributions ${ }^{6}$

$$
F(r):=P\left(x \in \mathbb{R}^{n} \mid\|x\| \leq r\right) \text { for all } r \in[0, \infty) .
$$

Definition 2.1. For $\kappa \in(-1, \infty)$ we define a specific member of the rotationally symmetric $\kappa$-distribution family by its radial distribution

$$
F_{\kappa}(r):= \begin{cases}\frac{\int_{0}^{r}\left(1-\tau^{2}\right)^{\kappa} \tau^{n-1} d \tau}{\int_{0}^{1}\left(1-\tau^{2}\right)^{\kappa} \tau^{n-1} d \tau} & \text { for } 0 \leq r \leq 1 \\ 1 & \text { for } r>1\end{cases}
$$

with density function

$$
f_{\kappa}(r):= \begin{cases}\frac{\left(1-r^{2}\right)^{\kappa} r^{n-1}}{\int_{0}^{1}\left(1-\tau^{2}\right)^{\kappa} \tau^{n-1} d \tau} & \text { for } 0 \leq r \leq 1 \\ 0 & \text { for } r>1\end{cases}
$$

so that $F_{\kappa}(r)=\int_{0}^{r} f_{\kappa}(\tau) d \tau$ holds for all $r \in[0, \infty)$.

The distribution parameter $\kappa$ specifies the distribution of the distance of the vectors to the origin. Here, the weight will be close to the origin, when $\kappa$ is large, and apart from 0 , when $\kappa$ is small. The following special cases are particularly interesting:

$\kappa \rightarrow-1 \widehat{=}$ Uniform distribution on the unit sphere (surface of the unit ball).

$\kappa=0 \hat{=}$ Uniform distribution on the (whole) unit ball.

We will use this distribution family as rotationally symmetric distribution for the first $n$ coordinates of our vectors, while the last coordinate still shall be distributed independently uniformly on $[-1,1]$ according to our augmented rotation-symmetry-model.

\subsection{Partition of the analysis}

In order to get a sharp upper bound we start by splitting the integral into

$$
\mathbb{E}_{m, n}[\tilde{S}]=I_{A}+I_{B}+I_{C}
$$

\footnotetext{
${ }^{6}$ Compare Borgwardt [2].
} 
where

$$
\begin{aligned}
I_{A}=\left(\begin{array}{c}
m \\
n+1
\end{array}\right) \cdot(n+1) & \cdot \lambda_{n-1}\left(\omega_{n}\right) \cdot \\
& \cdot \int_{0}^{\frac{\pi}{2}} \int_{|\sin (\varphi)-\cos (\varphi)|}^{\sin (\varphi)+\cos (\varphi)} G(h, \varphi)^{m-(n+1)} \cdot \frac{(\sin \varphi)^{n-1}}{(\cos \varphi)^{n+2}} \cdot \Lambda(h, \varphi) \cdot d h d \varphi
\end{aligned}
$$

and

$$
\begin{aligned}
I_{B}=\left(\begin{array}{c}
m \\
n+1
\end{array}\right) \cdot(n+1) & \cdot \lambda_{n-1}\left(\omega_{n}\right) \cdot \\
& \cdot \int_{0}^{\frac{\pi}{4}} \int_{0}^{|\sin (\varphi)-\cos (\varphi)|} G(h, \varphi)^{m-(n+1)} \cdot \frac{(\sin \varphi)^{n-1}}{(\cos \varphi)^{n+2}} \cdot \Lambda(h, \varphi) \cdot d h d \varphi
\end{aligned}
$$

as well as

$$
\begin{aligned}
I_{C}=\left(\begin{array}{c}
m \\
n+1
\end{array}\right) \cdot(n+1) & \cdot \lambda_{n-1}\left(\omega_{n}\right) . \\
& \cdot \int_{\frac{\pi}{4}}^{\frac{\pi}{2}} \int_{0}^{|\sin (\varphi)-\cos (\varphi)|} G(h, \varphi)^{m-(n+1)} \cdot \frac{(\sin \varphi)^{n-1}}{(\cos \varphi)^{n+2}} \cdot \Lambda(h, \varphi) \cdot d h d \varphi,
\end{aligned}
$$

so $I_{A}$ counts facets of category $\mathrm{A}, I_{B}$ facets of category B and $I_{C}$ facets of category $\mathrm{C}$. $I_{B}$ is asymptotically harmless as we can bound it by ${ }^{7}$

$$
I_{B} \leq 2^{n+1} \cdot \Lambda_{n} \cdot \lambda_{n}\left(\Omega_{n}\right) \leq \frac{(4 \pi)^{\frac{n}{2}}}{2 \Gamma\left(\frac{n+2}{2}\right)}
$$

where $\Gamma(\cdot)$ is the well-known Gamma function and

$$
\Lambda_{n}:=\frac{1}{2^{n+1}} \cdot \int_{\mathbb{R}^{n}} \ldots \int_{\mathbb{R}^{n}}|\operatorname{det} B| \cdot W\left(b_{1}, \ldots, b_{n}\right) \cdot f\left(b_{1}\right) \ldots f\left(b_{n+1}\right) \cdot d b_{1} \ldots d b_{n+1} .
$$

For the analysis of $I_{A}$ and $I_{C}$ we perform another transformation of coordinates. We shall describe these integrals no longer by $h$ and $\varphi$, but by $z$ and $s$. By use of that quantities $I_{A}$ and $I_{C}$ will be split into

$$
I_{A}=J_{A}^{z>1}+J_{A}^{z \leq 1}
$$

where

$$
J_{A}^{z>1}=\left(\begin{array}{c}
m \\
n+1
\end{array}\right) \cdot(n+1) \cdot \lambda_{n-1}\left(\omega_{n}\right) \cdot \int_{1}^{2} \int_{0}^{2} G(s, z)^{m-(n+1)} \cdot \frac{s^{n}}{z^{n+1}} \cdot \Lambda(z) \cdot d s d z
$$

\footnotetext{
${ }^{7}$ See $[8]$ for a proof.
} 
and

$$
J_{A}^{z \leq 1}=\left(\begin{array}{c}
m \\
n+1
\end{array}\right) \cdot(n+1) \cdot \lambda_{n-1}\left(\omega_{n}\right) \cdot \int_{0}^{1} \int_{0}^{2} G(s, z)^{m-(n+1)} \cdot \frac{s^{n}}{z^{n+1}} \cdot \Lambda(z) \cdot d s d z
$$

respectively

$$
I_{C}=J_{C}^{z>1}+J_{C}^{z \leq 1}
$$

where

$$
J_{C}^{z>1}=\left(\begin{array}{c}
m \\
n+1
\end{array}\right) \cdot(n+1) \cdot \lambda_{n-1}\left(\omega_{n}\right) \cdot \int_{1}^{2} \int_{2}^{\infty} G(s, z)^{m-(n+1)} \cdot \frac{s^{n}}{z^{n+1}} \cdot \Lambda(s, z) \cdot d s d z
$$

and

$$
J_{C}^{z \leq 1}=\left(\begin{array}{c}
m \\
n+1
\end{array}\right) \cdot(n+1) \cdot \lambda_{n-1}\left(\omega_{n}\right) \cdot \int_{0}^{1} \int_{2}^{\infty} G(s, z)^{m-(n+1)} \cdot \frac{s^{n}}{z^{n+1}} \cdot \Lambda(s, z) \cdot d s d z
$$

For $J_{A}^{z>1}$ and $J_{C}^{z>1}$ we have the constant upper bound ${ }^{8}$

$$
5 \cdot C(F, n)^{-n} \cdot \Lambda_{n} \cdot \lambda_{n}\left(\Omega_{n}\right) .
$$

The accurate analysis of $J_{A}^{z \leq 1}$ and $J_{C}^{z \leq 1}$ will be the main part of this section.

\subsection{Estimation of the spherical measure}

An essential part in the integral $(1.1)$ is the term $\Lambda(h, \varphi)$. So when we want to get a good upper bound for the whole integral, we are forced to analyze this term first. It is obvious that the expression $\Lambda(h, \varphi)$ mainly depends on $|\operatorname{det} B|$ and $W\left(b_{1}, \ldots, b_{n}\right)$. In order to simplify the analysis we want to observe both influences separately. Therefore we use the inequality of Cauchy-Schwarz, which yields

$$
\Lambda(h, \varphi) \leq \sqrt{\Lambda_{B}(h, \varphi) \cdot \Lambda_{W}(h, \varphi)}
$$

where

$$
\Lambda_{B}(h, \varphi):=\int_{\mathbb{R}^{n}} \ldots \int_{\mathbb{R}^{n}}|\operatorname{det} B|^{2} \cdot \tilde{f}\left(\tilde{b}_{1}\right) \ldots \tilde{f}\left(\tilde{b}_{n+1}\right) \cdot d b_{1} \ldots d b_{n+1}
$$

and

$$
\Lambda_{W}(h, \varphi):=\int_{\mathbb{R}^{n}} \ldots \int_{\mathbb{R}^{n}} W\left(b_{1}, \ldots, b_{n}\right)^{2} \cdot \tilde{f}\left(\tilde{b}_{1}\right) \ldots \tilde{f}\left(\tilde{b}_{n+1}\right) \cdot d b_{1} \ldots d b_{n+1} .
$$

${ }^{8}$ See [8] for a proof. 


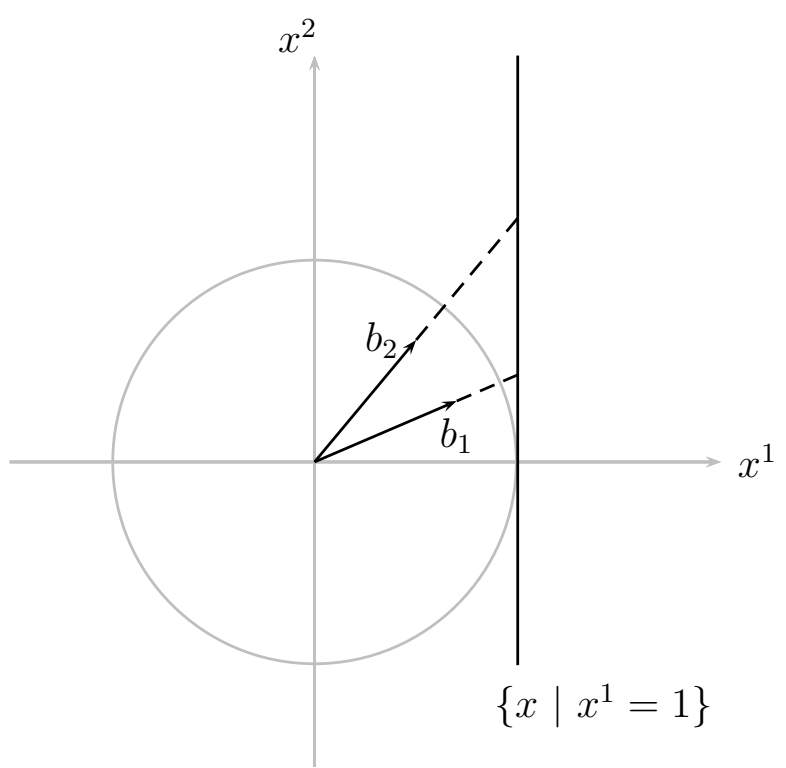

Figure 2.1: Illustration of the estimation of the spherical measure $W$ in the projection onto $\mathbb{R}^{n}$ for $n=2$.

The spherical measure $W\left(b_{1}, \ldots, b_{n}\right)$ can be bounded trivially by

$$
W\left(b_{1}, \ldots, b_{n}\right) \leq \frac{1}{2}
$$

which is a very simple upper bound, but of course too rough for a precise asymptotic analysis. A much more accurate estimation is

$$
\begin{aligned}
W\left(b_{1}, \ldots, b_{n}\right) \leq \frac{1}{\lambda_{n}\left(\Omega_{n}\right)} \cdot \lambda_{n}\left(\operatorname{conv}\left(0, \frac{1}{b_{1}^{1}} b_{1}, \ldots, \frac{1}{b_{n}^{1}} b_{n}\right)\right) \\
=\frac{1}{\lambda_{n}\left(\Omega_{n}\right)} \cdot \frac{1}{b_{1}^{1} \ldots b_{n}^{1}} \cdot \lambda_{n}\left(\operatorname{conv}\left(0, b_{1}, \ldots, b_{n}\right)\right) \\
=\frac{1}{\lambda_{n}\left(\Omega_{n}\right)} \cdot \frac{1}{b_{1}^{1} \ldots b_{n}^{1}} \cdot \frac{1}{n !} \cdot\left|\operatorname{det}\left(b_{1}, \ldots, b_{n}\right)\right| \\
\quad \leq \frac{1}{\lambda_{n}\left(\Omega_{n}\right)} \cdot \frac{(\sin \varphi)^{n}}{(h-\cos \varphi)^{n}} \cdot \frac{1}{n !} \cdot\left|\operatorname{det}\left(b_{1}, \ldots, b_{n}\right)\right|,
\end{aligned}
$$

which holds for $h \geq \cos (\varphi)$ respectively $z \leq 1 .{ }^{9}$ Recalling

$$
G(h, \varphi)=\int_{-\infty}^{\infty} \ldots \int_{-\infty}^{\infty} \int_{-\infty}^{\frac{h-x^{1} \sin (\varphi)}{\cos (\varphi)}} f(x) \cdot \hat{f}\left(\tilde{x}^{n+1}\right) \cdot d \tilde{x}^{n+1} d \tilde{x}^{n} \ldots d \tilde{x}^{1}
$$

\footnotetext{
${ }^{9}$ See $[8]$ for details.
} 
and defining

$$
\begin{aligned}
g(h, \varphi) & :=\frac{1}{\cos (\varphi)} \cdot \int_{-\infty}^{\infty} \ldots \int_{-\infty}^{\infty} f(x) \cdot \hat{f}\left(\frac{h-x^{1} \sin (\varphi)}{\cos (\varphi)}\right) \cdot d x^{1} \ldots d x^{n}, \\
g_{2}(h, \varphi) & :=\frac{1}{\cos (\varphi)} \cdot \int_{-\infty}^{\infty} \ldots \int_{-\infty}^{\infty}\left(x^{1}\right)^{2} \cdot f(x) \cdot \hat{f}\left(\frac{h-x^{1} \sin (\varphi)}{\cos (\varphi)}\right) \cdot d x^{1} \ldots d x^{n}, \\
g_{3}(h, \varphi) & :=\frac{1}{\cos (\varphi)} \cdot \int_{-\infty}^{\infty} \ldots \int_{-\infty}^{\infty}\left(x^{2}\right)^{2} \cdot f(x) \cdot \hat{f}\left(\frac{h-x^{1} \sin (\varphi)}{\cos (\varphi)}\right) \cdot d x^{1} \ldots d x^{n}, \\
g_{4}(h, \varphi) & :=\frac{1}{\cos (\varphi)} \cdot \int_{-\infty}^{\infty} \ldots \int_{-\infty}^{\infty} x^{1} \cdot f(x) \cdot \hat{f}\left(\frac{h-x^{1} \sin (\varphi)}{\cos (\varphi)}\right) \cdot d x^{1} \ldots d x^{n}
\end{aligned}
$$

where $g(h, \varphi)$ is the partial derivation of $G(h, \varphi)$ with respect to $h$, we obtain the following relations. ${ }^{10}$

\section{Lemma 2.2.}

$$
\Lambda_{B}(h, \varphi)=(n+1) ! \cdot(\cos \varphi)^{n+1} \cdot g_{3}(h, \varphi)^{n-1} \cdot\left[g(h, \varphi) g_{2}(h, \varphi)-g_{4}(h, \varphi)^{2}\right]
$$

and for $h \geq \cos (\varphi)$

$$
\Lambda_{W}(h, \varphi) \leq \frac{1}{\left(\lambda_{n}\left(\Omega_{n}\right)\right)^{2} n !} \cdot \frac{(\sin \varphi)^{2 n}}{(h-\cos \varphi)^{2 n}} \cdot(\cos \varphi)^{n+1} \cdot g(h, \varphi) \cdot g_{2}(h, \varphi) \cdot g_{3}(h, \varphi)^{n-1} .
$$

\subsection{Analysis of category A}

We now come to the precise analysis of the category A. We know from [8] that the main part of the integral can be described in the Cartesian coordinates $z$ and $s$ in the form

$$
J_{A}^{z \leq 1}=\left(\begin{array}{c}
m \\
n+1
\end{array}\right) \cdot(n+1) \cdot \lambda_{n-1}\left(\omega_{n}\right) \cdot \int_{0}^{1} \int_{0}^{2} G(s, z)^{m-(n+1)} \cdot \frac{s^{n}}{z^{n+1}} \cdot \Lambda(z) \cdot d s d z
$$

where

$$
G(s, z)=\int_{-\infty}^{\infty} \ldots \int_{-\infty}^{\infty} \int_{-\infty}^{\frac{(1-z) s+z-x^{1} s}{z}} f(x) \cdot \hat{f}\left(\tilde{x}^{n+1}\right) \cdot d \tilde{x}^{n+1} d \tilde{x}^{n} \ldots d \tilde{x}^{1}
$$

10 See [8] for a proof. 
and

$$
\begin{aligned}
\Lambda(z)=\int_{1-z}^{1} \int_{-\infty}^{\infty} \ldots & \int_{-\infty}^{\infty} \ldots \int_{1-z}^{1} \int_{-\infty}^{\infty} \ldots \int_{-\infty}^{\infty}|\operatorname{det} B| \cdot W\left(b_{1}, \ldots, b_{n}\right) . \\
& \cdot f\left(b_{1}\right) \ldots f\left(b_{n+1}\right) \cdot \frac{1}{2} \ldots \frac{1}{2} \cdot d b_{1}^{n} \ldots d b_{1}^{2} d b_{1}^{1} \ldots d b_{n+1}^{n} \ldots d b_{n+1}^{2} d b_{n+1}^{1} .
\end{aligned}
$$

It is remarkable that this term $\Lambda$ depends on $z$ only and not on $s$. This will make the calculations easier than in the case of category $\mathrm{C}$ where $\Lambda$ depends both on $z$ and $s$. Using the adapted notation

$$
\begin{aligned}
\Lambda_{B}(z)=\int_{1-z}^{1} \int_{-\infty}^{\infty} \ldots & \int_{-\infty}^{\infty} \ldots \int_{1-z}^{1} \int_{-\infty}^{\infty} \ldots \int_{-\infty}^{\infty}|\operatorname{det} B|^{2} . \\
& \cdot f\left(b_{1}\right) \ldots f\left(b_{n+1}\right) \cdot \frac{1}{2} \ldots \frac{1}{2} \cdot d b_{1}^{n} \ldots d b_{1}^{2} d b_{1}^{1} \ldots d b_{n+1}^{n} \ldots d b_{n+1}^{2} d b_{n+1}^{1}
\end{aligned}
$$

and

$$
\begin{aligned}
\Lambda_{W}(z)=\int_{1-z}^{1} \int_{-\infty}^{\infty} \ldots & \int_{-\infty}^{\infty} \ldots \int_{1-z}^{1} \int_{-\infty}^{\infty} \ldots \int_{-\infty}^{\infty} W\left(b_{1}, \ldots, b_{n}\right)^{2} . \\
& \cdot f\left(b_{1}\right) \ldots f\left(b_{n+1}\right) \cdot \frac{1}{2} \ldots \frac{1}{2} \cdot d b_{1}^{n} \ldots d b_{1}^{2} d b_{1}^{1} \ldots d b_{n+1}^{n} \ldots d b_{n+1}^{2} d b_{n+1}^{1}
\end{aligned}
$$

as well as

$$
\begin{aligned}
& g(z):=\frac{1}{2 z} \cdot \int_{1-z}^{1} \int_{-\infty}^{\infty} \ldots \int_{-\infty}^{\infty} f\left(x^{1}, \ldots, x^{n}\right) \cdot d x^{n} \ldots d x^{2} d x^{1}, \\
& g_{2}(z):=\frac{1}{2 z} \cdot \int_{1-z}^{1} \int_{-\infty}^{\infty} \ldots \int_{-\infty}^{\infty}\left(x^{1}\right)^{2} \cdot f\left(x^{1}, \ldots, x^{n}\right) \cdot d x^{n} \ldots d x^{2} d x^{1}, \\
& g_{3}(z):=\frac{1}{2 z} \cdot \int_{1-z}^{1} \int_{-\infty}^{\infty} \ldots \int_{-\infty}^{\infty}\left(x^{2}\right)^{2} \cdot f\left(x^{1}, \ldots, x^{n}\right) \cdot d x^{n} \ldots d x^{2} d x^{1}, \\
& g_{4}(z):=\frac{1}{2 z} \cdot \int_{1-z}^{1} \int_{-\infty}^{\infty} \ldots \int_{-\infty}^{\infty} x^{1} \cdot f\left(x^{1}, \ldots, x^{n}\right) \cdot d x^{n} \ldots d x^{2} d x^{1}
\end{aligned}
$$

we obtain the following Lemma.

\section{Lemma 2.3.}

$$
\Lambda_{B}(z)=(n+1) ! \cdot z^{n+1} \cdot g_{3}(z)^{n-1} \cdot\left[g(z) g_{2}(z)-g_{4}(z)^{2}\right]
$$


and for $z \leq 1$

$$
\Lambda_{W}(z) \leq \frac{1}{\left(\lambda_{n}\left(\Omega_{n}\right)\right)^{2} n !} \cdot \frac{1}{(1-z)^{2 n}} \cdot z^{n+1} \cdot g(z) \cdot g_{2}(z) \cdot g_{3}(z)^{n-1} .
$$

From the inequality of Cauchy-Schwarz we get a first estimation for $J_{A}^{z \leq 1}$.

Theorem 2.4. For any $q \in(0,1)$ holds

$$
\begin{aligned}
& J_{A}^{z \leq 1} \leq\left(\begin{array}{c}
m \\
n+1
\end{array}\right) \cdot(n+1) \cdot \lambda_{n-1}\left(\omega_{n}\right) \cdot \int_{q}^{1} \int_{0}^{2} G(s, z)^{m-(n+1)} \cdot \frac{s^{n}}{z^{n+1}} \cdot \Lambda(z) \cdot d s d z+ \\
&+\left(\begin{array}{c}
m \\
n+1
\end{array}\right) \cdot(n+1)^{\frac{3}{2}} \cdot \frac{\lambda_{n-1}\left(\omega_{n}\right)}{\lambda_{n}\left(\Omega_{n}\right)} \cdot \int_{0}^{q} \int_{0}^{2} G(s, z)^{m-(n+1)} \cdot \frac{s^{n}}{(1-z)^{n}} \cdot \\
& \cdot g_{3}(z)^{n-1} \cdot \sqrt{g(z) g_{2}(z)\left[g(z) g_{2}(z)-g_{4}(z)^{2}\right]} \cdot d s d z .
\end{aligned}
$$

For the following calculations we observe some relations between the functions $G$ and $g, g_{2}, g_{3}, g_{4}$, which can also be read in [8].

Lemma 2.5. For $s \leq 2$ we have

$$
G(s, z)=1-\left[g_{4}(z)-(1-z) g(z)\right] \cdot s .
$$

The partial derivation of $G(s, z)$ with respect to $z$ is given by

$$
\frac{\partial G}{\partial z}(s, z)=\frac{g_{4}(z)-g(z)}{z} \cdot s .
$$

We define

$$
\Phi(s, z):=1-G(s, z)
$$

and state

$$
\begin{gathered}
g(z)=\frac{1}{2 z} \cdot \frac{\Gamma\left(\kappa+1+\frac{n}{2}\right)}{\sqrt{\pi} \Gamma\left(\kappa+1+\frac{n-1}{2}\right)} \cdot \int_{1-z}^{1}\left(1-\zeta^{2}\right)^{\frac{n-1}{2}+\kappa} d \zeta, \\
g_{2}(z)=\frac{1}{2 z} \cdot \frac{\Gamma\left(\kappa+1+\frac{n}{2}\right)}{\sqrt{\pi} \Gamma\left(\kappa+1+\frac{n-1}{2}\right)} \cdot \int_{1-z}^{1} \zeta^{2} \cdot\left(1-\zeta^{2}\right)^{\frac{n-1}{2}+\kappa} d \zeta, \\
g_{3}(z)=\frac{1}{2 z} \cdot \frac{\Gamma\left(\kappa+1+\frac{n}{2}\right)}{2 \sqrt{\pi} \Gamma\left(\kappa+1+\frac{n+1}{2}\right)} \cdot \int_{1-z}^{1}\left(1-\zeta^{2}\right)^{\frac{n+1}{2}+\kappa} d \zeta,
\end{gathered}
$$




$$
g_{4}(z)=\frac{1}{2 z} \cdot \frac{\Gamma\left(\kappa+1+\frac{n}{2}\right)}{\sqrt{\pi} \Gamma\left(\kappa+1+\frac{n-1}{2}\right)} \cdot \int_{1-z}^{1} \zeta \cdot\left(1-\zeta^{2}\right)^{\frac{n-1}{2}+\kappa} d \zeta
$$

for the case of our distribution family. A very important relation is the following Lemma.

Lemma 2.6. For $s \in(0,2)$ there is a function $\alpha:[0,1] \rightarrow \mathbb{R}$ with

$$
g(z) g_{2}(z)-g_{4}(z)^{2}=\left[\frac{\Phi(s, z)}{s}\right]^{2} \cdot \frac{n+1+2 \kappa}{n+5+2 \kappa} \cdot(1+\alpha(z))
$$

and $\alpha(z) \rightarrow 0$ for $z \rightarrow 0$.

Proof. By application of the rule of l'Hospital in three steps we get

$$
\begin{aligned}
& \lim _{z \rightarrow 0} \frac{g(z) g_{2}(z)-g_{4}(z)^{2}}{\left[2\left(\kappa+1+\frac{n-1}{2}\right)\right]^{2} g_{3}(z)^{2}} \\
& =\lim _{z \rightarrow 0} \frac{\int_{1-z}^{1}\left(1-\zeta^{2}\right)^{\frac{n-1}{2}+\kappa} d \zeta \int_{1-z}^{1} \zeta^{2}\left(1-\zeta^{2}\right)^{\frac{n-1}{2}+\kappa} d \zeta-\left[\int_{1-z}^{1} \zeta\left(1-\zeta^{2}\right)^{\frac{n-1}{2}+\kappa} d \zeta\right]^{2}}{\left[\int_{1-z}^{1}\left(1-\zeta^{2}\right)^{\frac{n+1}{2}+\kappa} d \zeta\right]^{2}} \\
& =\lim _{z \rightarrow 0}\left[\int_{1-z}^{1} \zeta^{2}\left(1-\zeta^{2}\right)^{\frac{n-1}{2}+\kappa} d \zeta+(1-z)^{2} \int_{1-z}^{1}\left(1-\zeta^{2}\right)^{\frac{n-1}{2}+\kappa} d \zeta-\right. \\
& \left.-2(1-z) \int_{1-z}^{1} \zeta\left(1-\zeta^{2}\right)^{\frac{n-1}{2}+\kappa} d \zeta\right] /\left[2(2-z) z \int_{1-z}^{1}\left(1-\zeta^{2}\right)^{\frac{n+1}{2}+\kappa} d \zeta\right] \\
& =\lim _{z \rightarrow 0}\left[2 \int_{1-z}^{1} \zeta\left(1-\zeta^{2}\right)^{\frac{n-1}{2}+\kappa} d \zeta-2(1-z) \int_{1-z}^{1}\left(1-\zeta^{2}\right)^{\frac{n-1}{2}+\kappa} d \zeta\right] / \\
& {\left[2(2-z)^{\frac{n+3}{2}+\kappa} z^{\frac{n+3}{2}+\kappa}+4(1-z) \int_{1-z}^{1}\left(1-\zeta^{2}\right)^{\frac{n+1}{2}+\kappa} d \zeta\right]} \\
& =\lim _{z \rightarrow 0}\left[2 \int_{1-z}^{1}\left(1-\zeta^{2}\right)^{\frac{n-1}{2}+\kappa} d \zeta\right] /\left[2\left(\frac{n+3}{2}+\kappa\right)(2-z)^{\frac{n+3}{2}+\kappa} z^{\frac{n+1}{2}+\kappa}-\right. \\
& -2\left(\frac{n+3}{2}+\kappa\right)(2-z)^{\frac{n+1}{2}+\kappa} z^{\frac{n+3}{2}+\kappa}+4(1-z)(2-z)^{\frac{n+1}{2}+\kappa} z^{\frac{n+1}{2}+\kappa}- \\
& \left.-4 \int_{1-z}^{1}\left(1-\zeta^{2}\right)^{\frac{n+1}{2}+\kappa} d \zeta\right] .
\end{aligned}
$$


Now the term in the numerator has an order of growth like $z^{\frac{n+1}{2}+\kappa}$. In the denominator there are two terms with an order of growth like $z^{\frac{n+1}{2}+\kappa}$ and two with $z^{\frac{n+3}{2}+\kappa}$. Dividing by $z^{\frac{n+1}{2}+\kappa}$ those with $z^{\frac{n+3}{2}+\kappa}$ in the denominator disappear, while the other terms yield a constant (in $n$ and $\kappa$ ), when $z$ is going to 0 . Taking into regard

$$
\lim _{z \rightarrow 0} \frac{2 \int_{1-z}^{1}\left(1-\zeta^{2}\right)^{\frac{n-1}{2}+\kappa} d \zeta}{z^{\frac{n+1}{2}+\kappa}}=\lim _{z \rightarrow 0} \frac{2(2-z)^{\frac{n-1}{2}+\kappa} z^{\frac{n-1}{2}+\kappa}}{\left(\frac{n+1}{2}+\kappa\right) z^{\frac{n-1}{2}+\kappa}}=\frac{2^{\frac{n+1}{2}+\kappa}}{\left(\frac{n+1}{2}+\kappa\right)}
$$

it follows

$$
\lim _{z \rightarrow 0} \frac{g(z) g_{2}(z)-g_{4}(z)^{2}}{\left[2\left(\kappa+1+\frac{n-1}{2}\right)\right]^{2} g_{3}(z)^{2}}=\frac{1}{4\left(\frac{n+1}{2}+\kappa\right)\left(\frac{n+5}{2}+\kappa\right)} .
$$

As we already know that a function $\beta:[0,1] \rightarrow \mathbb{R}$ exists with ${ }^{11}$

$$
g_{3}(z)=\left[\frac{\Phi(s, z)}{s}\right] \cdot(1+\beta(z))
$$

and $\beta(z) \rightarrow 0$ for $z \rightarrow 0$, we can state

$$
g(z) g_{2}(z)-g_{4}(z)^{2}=\left[\frac{\Phi(s, z)}{s}\right]^{2} \cdot \frac{n+1+2 \kappa}{n+5+2 \kappa} \cdot(1+\beta(z))^{2}
$$

and the claim is obvious by setting $(1+\alpha(z)):=(1+\beta(z))^{2}$.

Now we can formulate our main result for the category A.

Theorem 2.7. For $n \geq 3$ there is a function $\varepsilon_{\kappa}(m, n)$, which depends on the distribution parameter $\kappa$, with $\varepsilon_{\kappa}(m, n) \rightarrow 0$ for $m \rightarrow \infty, n$ constant and

$$
\begin{aligned}
& J_{A}^{z \leq 1}=\left(\begin{array}{c}
m \\
n+1
\end{array}\right) \cdot(n+1) \cdot \lambda_{n-1}\left(\omega_{n}\right) \cdot \int_{0}^{1} \int_{0}^{2} G(s, z)^{m-(n+1)} \cdot \frac{s^{n}}{z^{n+1}} \cdot \Lambda(z) \cdot d s d z \\
& \leq \ln (m) \cdot(n+1)^{\frac{3}{2}} \cdot(n+3+2 \kappa)^{\frac{2}{n+1+2 \kappa}} \cdot\left(1+\varepsilon_{\kappa}(m, n)\right) .
\end{aligned}
$$

Proof. Using the notation

$$
A(z):=g_{4}(z)-(1-z) g(z)
$$

first we define for $q \in(0,1)$

$$
J_{A}^{1}:=\left(\begin{array}{c}
m \\
n+1
\end{array}\right) \cdot(n+1) \cdot \lambda_{n-1}\left(\omega_{n}\right) \cdot \int_{0}^{q} \int_{\frac{1}{m}}^{2} G(s, z)^{m-(n+1)} \cdot \frac{s^{n}}{z^{n+1}} \cdot \Lambda(z) \cdot d s d z
$$

11 See [8] for a proof. 
and

$$
J_{A}^{2}:=\left(\begin{array}{c}
m \\
n+1
\end{array}\right) \cdot(n+1) \cdot \lambda_{n-1}\left(\omega_{n}\right) \cdot \int_{0}^{1} \int_{0}^{\frac{1}{m}} G(s, z)^{m-(n+1)} \cdot \frac{s^{n}}{z^{n+1}} \cdot \Lambda(z) \cdot d s d z
$$

as well as

$$
J_{A}^{3}:=\left(\begin{array}{c}
m \\
n+1
\end{array}\right) \cdot(n+1) \cdot \lambda_{n-1}\left(\omega_{n}\right) \cdot \int_{q}^{1} \int_{\frac{1}{m}}^{2} G(s, z)^{m-(n+1)} \cdot \frac{s^{n}}{z^{n+1}} \cdot \Lambda(z) \cdot d s d z
$$

so that obviously $J_{A}^{z \leq 1}=J_{A}^{1}+J_{A}^{2}+J_{A}^{3}$. The integral $J_{A}^{3}$ is asymptotically harmless. If we choose $q>0$ small enough, i. e. if we choose it such that $2 A(q) \leq 1$ holds, we can estimate

$$
\begin{aligned}
& J_{A}^{3}=\left(\begin{array}{c}
m \\
n+1
\end{array}\right) \cdot(n+1) \cdot \lambda_{n-1}\left(\omega_{n}\right) \cdot \int_{q}^{1} \int_{\frac{1}{m}}^{2} G(s, z)^{m-(n+1)} \cdot \frac{s^{n}}{z^{n+1}} \cdot \Lambda(z) \cdot d s d z \\
& \leq\left(\begin{array}{c}
m \\
n+1
\end{array}\right) \cdot(n+1) \cdot \lambda_{n-1}\left(\omega_{n}\right) \cdot \int_{q}^{1} \int_{0}^{2}[1-A(q) s]^{m-(n+1)} \cdot \frac{s^{n}}{z^{n+1}} \cdot \Lambda(z) \cdot d s d z
\end{aligned}
$$

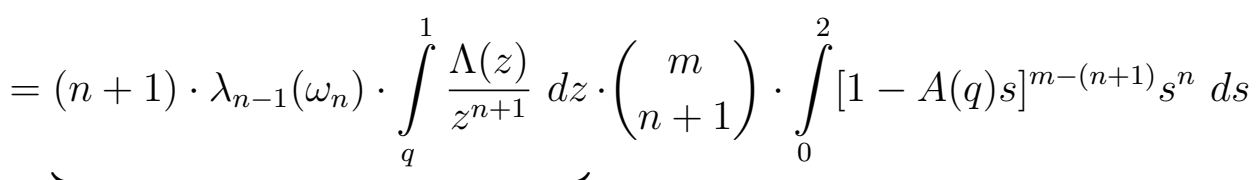

$$
\begin{aligned}
& =: \tilde{C}(\kappa, n) \\
& \stackrel{2 A(q)}{\leq} \leq 1 \quad \tilde{C}(\kappa, n) \cdot \frac{1}{A(q)^{n+1}} \cdot\left(\begin{array}{c}
m \\
n+1
\end{array}\right) \cdot \int_{0}^{1}(1-u)^{m-(n+1)} u^{n} d u \\
& =\tilde{C}(\kappa, n) \cdot \frac{1}{A(q)^{n+1}} \cdot\left(\begin{array}{c}
m \\
n+1
\end{array}\right) \cdot \frac{\Gamma(n+1) \Gamma(m-n)}{\Gamma(m+1)} \\
& =\tilde{C}(\kappa, n) \cdot \frac{1}{A(q)^{n+1}} \cdot \frac{m ! n !(m-n-1) !}{(m-n-1) !(n+1) ! m !}=\tilde{C}(\kappa, n) \cdot \frac{1}{A(q)^{n+1}} \cdot \frac{1}{n+1}=: C_{3}(\kappa, n)
\end{aligned}
$$

where $C_{3}(\kappa, n)$ is a constant depending only on the dimension $n$ and the distribution parameter $\kappa$, but not on $m$. The integral $J_{A}^{2}$ also can be estimated by a constant independent of $m$. For this purpose we use the simple fact that the hyperplane can cut the cylinder barrel in a distance lower than $\frac{1}{m}$ to the cover only, if all points $\tilde{a}_{1}, \ldots, \tilde{a}_{n+1}$ are located in this region. Because if one point would have a greater distance to the cylinder cover, the hyperplane would cut the barrel in a greater distance to the cover, too. So as a result of our stochastic model we can roughly estimate

$$
J_{A}^{2} \leq\left(\begin{array}{c}
m \\
n+1
\end{array}\right) \cdot\left(\frac{1}{m}\right)^{n+1} \leq m^{n+1} \cdot\left(\frac{1}{m}\right)^{n+1}=1 .
$$


Now we care about $J_{A}^{1}$. For $q \leq 1$ it results from Theorem 2.4 and the obvious fact $g_{2}(z) \leq g(z)$ that

$$
\begin{gathered}
J_{A}^{1} \leq\left(\begin{array}{c}
m \\
n+1
\end{array}\right) \cdot(n+1)^{\frac{3}{2}} \cdot \frac{\lambda_{n-1}\left(\omega_{n}\right)}{\lambda_{n}\left(\Omega_{n}\right)} \cdot \int_{0}^{q} \int_{\frac{1}{m}}^{2} G(s, z)^{m-(n+1)} \cdot \frac{s^{n}}{(1-z)^{n}} \cdot \\
\cdot \frac{g(z)-g_{4}(z)}{z} \cdot g_{3}(z)^{n-1} \cdot z \cdot \frac{\sqrt{g(z) g_{2}(z)\left[g(z) g_{2}(z)-g_{4}(z)^{2}\right]}}{g(z)-g_{4}(z)} \cdot d s d z \\
\leq\left(\begin{array}{c}
m \\
n+1
\end{array}\right) \cdot \frac{(n+1)^{\frac{3}{2}}}{(1-q)^{n}} \cdot \frac{\lambda_{n-1}\left(\omega_{n}\right)}{\lambda_{n}\left(\Omega_{n}\right)} \cdot \int_{0}^{q} \int_{\frac{1}{m}}^{2} G(s, z)^{m-(n+1)} \cdot s^{n} \cdot \\
\cdot \frac{g(z)-g_{4}(z)}{z} \cdot g_{3}(z)^{n-1} \cdot g(z) \cdot z \cdot \frac{\sqrt{g(z) g_{2}(z)-g_{4}(z)^{2}}}{g(z)-g_{4}(z)} \cdot d s d z .
\end{gathered}
$$

From [8] and Lemma 2.6 we know that for any $\delta>0$ we can choose $q>0$ depending on the dimension $n$ and the distribution parameter $\kappa$ small enough so that for any $z \in[0, q]$ holds

1. $g_{3}(z) \leq \frac{\Phi(s, z)}{s} \cdot(1+\delta)$,

2. $g(z) \leq\left[\frac{\Phi(s, z)}{s}\right]^{\frac{n-1+2 \kappa}{n+1+2 \kappa}} \cdot\left[\kappa+1+\frac{n-1}{2}\right]^{\frac{n-1+2 \kappa}{n+1+2 \kappa}} \cdot\left[\frac{\Gamma\left(\kappa+1+\frac{n}{2}\right)}{2 \sqrt{\pi} \Gamma\left(\kappa+1+\frac{n-1}{2}\right)}\right]^{\frac{2}{n+1+2 \kappa}}$. $\cdot(1+\delta)$

3. $\frac{1}{(1-q)^{n}} \leq 1+\delta$,

4. $\sqrt{g(z) g_{2}(z)-g_{4}(z)^{2}} \leq \frac{\Phi(s, z)}{s} \cdot \sqrt{\frac{n+1+2 \kappa}{n+5+2 \kappa}} \cdot(1+\delta)$,

5. $g(z)-g_{4}(z) \geq \frac{\Phi(s, z)}{s} \cdot\left(\frac{n+1}{2}+\kappa\right) \cdot(1+\delta)^{-1}$,

6. $z \leq\left[\frac{\Phi(s, z)}{s}\right]^{\frac{2}{n+1+2 \kappa}} \cdot\left[\frac{(n+3+2 \kappa)(n+1+2 \kappa) \sqrt{\pi} \Gamma\left(\kappa+1+\frac{n-1}{2}\right)}{\Gamma\left(\kappa+1+\frac{n}{2}\right) 2^{\frac{n+1}{2}+\kappa}}\right]^{\frac{2}{n+1+2 \kappa}}$. $\cdot(1+\delta)$ 
So for a suitable $q$ we get

$$
\begin{aligned}
& J_{A}^{1} \leq\left(\begin{array}{c}
m \\
n+1
\end{array}\right) \cdot(n+1)^{\frac{3}{2}} \cdot \frac{\lambda_{n-1}\left(\omega_{n}\right)}{2 \lambda_{n}\left(\Omega_{n}\right)} \cdot(n+3+2 \kappa)^{\frac{2}{n+1+2 \kappa}} . \\
& \cdot \int_{\frac{1}{m}}^{2} \int_{0}^{q}[1-\Phi(s, z)]^{m-(n+1)} \cdot \Phi(s, z)^{n-1} \cdot\left[\frac{\Phi(s, z)}{s}\right]^{\frac{n-1+2 \kappa}{n+1+2 \kappa}} \cdot \\
& \cdot\left[\frac{\Phi(s, z)}{s}\right]^{\frac{2}{n+1+2 \kappa}} \cdot \frac{\left[g(z)-g_{4}(z)\right] s}{z} \cdot d z d s \cdot(1+\delta)^{n+4} \\
& \leq\left(\begin{array}{c}
m \\
n+1
\end{array}\right) \cdot(n+1)^{\frac{3}{2}} \cdot \frac{\lambda_{n-1}\left(\omega_{n}\right)}{2 \lambda_{n}\left(\Omega_{n}\right)} \cdot(n+3+2 \kappa)^{\frac{2}{n+1+2 \kappa}} \cdot \\
& \cdot \int_{0}^{1}(1-\Phi)^{m-(n+1)} \cdot \Phi^{(n+1)-1} d \Phi \cdot \int_{\frac{1}{m}}^{\frac{1}{s}} d s \cdot(1+\delta)^{n+4} .
\end{aligned}
$$

In the last step we have performed the transformation $(s, \Phi) \mapsto(s, \Phi(s, z))$ with determinant of the Jacobian

$$
\operatorname{det}\left(\begin{array}{cc}
1 & 0 \\
\frac{\partial \Phi}{\partial s}(s, z) & \frac{\partial \Phi}{\partial z}(s, z)
\end{array}\right)=\frac{\partial \Phi}{\partial z}(s, z) \stackrel{\text { Lemma } 2.5}{=} \frac{\left[g(z)-g_{4}(z)\right] s}{z} .
$$

This transformation yields

$$
\begin{aligned}
\int_{\frac{1}{m}}^{2} \frac{1}{s} \int_{0}^{q}[1-\Phi(s, z)]^{m-(n+1)} \cdot \Phi(s, z)^{(n+1)-1} & \cdot \frac{\left[g(z)-g_{4}(z)\right] s}{z} d z d s \\
& =\int_{\frac{1}{m}}^{2} \frac{1}{s} \int_{0}^{\Phi(s, q)}[1-\Phi]^{m-(n+1)} \cdot \Phi^{(n+1)-1} d \Phi d s \\
& \leq \int_{\frac{1}{m}}^{2} \frac{1}{s} d s \cdot \int_{0}^{1}[1-\Phi]^{m-(n+1)} \cdot \Phi^{(n+1)-1} d \Phi .
\end{aligned}
$$

Now, we use the known equations, which can be found in the appendix of [4],

$$
\begin{aligned}
& \text { 1. } \frac{\lambda_{n-1}\left(\omega_{n}\right)}{\lambda_{n}\left(\Omega_{n}\right)}=n, \\
& \text { 2. }\left(\begin{array}{c}
m \\
n+1
\end{array}\right) \cdot \int_{0}^{1}(1-\Phi)^{m-(n+1)} \cdot \Phi^{(n+1)-1} d \Phi=\frac{1}{n+1} .
\end{aligned}
$$


Taking into regard

$$
\int_{\frac{1}{m}}^{2} \frac{1}{s} d s=[\ln (s)]_{\frac{1}{m}}^{2}=\ln (m)+\ln (2) \leq 2 \ln (m)
$$

we obtain

$$
J_{A}^{1} \leq \ln (m) \cdot(n+1)^{\frac{3}{2}} \cdot(n+3+2 \kappa)^{\frac{2}{n+1+2 \kappa}} \cdot(1+\delta)^{n+4} .
$$

For $m$ big enough we have $J_{A}^{2} \leq J_{A}^{1}$ and $J_{A}^{3} \leq J_{A}^{1}$. As we can choose for any $\varepsilon>0$ a $\delta>0$ with

$$
3(1+\delta)^{n+4} \leq 1+\varepsilon
$$

it finally results

$$
J_{A}^{z \leq 1} \leq \ln (m) \cdot(n+1)^{\frac{3}{2}} \cdot(n+3+2 \kappa)^{\frac{2}{n+1+2 \kappa}} \cdot\left(1+\varepsilon_{\kappa}(m, n)\right)
$$

and our claim is proven.

\subsection{Analysis of category C}

Now we focus on category C. Here, we know from [8]

$$
J_{C}^{z \leq 1}=\left(\begin{array}{c}
m \\
n+1
\end{array}\right) \cdot(n+1) \cdot \lambda_{n-1}\left(\omega_{n}\right) \cdot \int_{0}^{1} \int_{2}^{\infty} G(s, z)^{m-(n+1)} \cdot \frac{s^{n}}{z^{n+1}} \cdot \Lambda(s, z) \cdot d s d z
$$

where

$$
G(s, z)=\int_{-\infty}^{\infty} \ldots \int_{-\infty}^{\infty} \int_{-\infty}^{\frac{(1-z) s+z-x^{1} s}{z}} f(x) \cdot \hat{f}\left(\tilde{x}^{n+1}\right) \cdot d \tilde{x}^{n+1} d \tilde{x}^{n} \ldots d \tilde{x}^{1}
$$

and

$$
\begin{aligned}
\Lambda(s, z)= & \int_{1-z}^{1-\frac{z(s-2)}{s}} \int_{-\infty}^{\infty} \ldots \int_{-\infty}^{\infty} \ldots \int_{1-z}^{1-\frac{z(s-2)}{s}} \int_{-\infty}^{\infty} \ldots \int_{-\infty}^{\infty}|\operatorname{det} B| \cdot W\left(b_{1}, \ldots, b_{n}\right) . \\
& \cdot f\left(b_{1}\right) \ldots f\left(b_{n+1}\right) \cdot \frac{1}{2} \ldots \frac{1}{2} \cdot d b_{1}^{n} \ldots d b_{1}^{2} d b_{1}^{1} \ldots d b_{n+1}^{n} \ldots d b_{n+1}^{2} d b_{n+1}^{1} .
\end{aligned}
$$

Analogously to category A we note

$$
\begin{aligned}
\Lambda_{B}(s, z)= & \int_{1-z}^{1-\frac{z(s-2)}{s}} \int_{-\infty}^{\infty} \ldots \int_{-\infty}^{\infty} \ldots \int_{1-z}^{1-\frac{z(s-2)}{s}} \int_{-\infty}^{\infty} \ldots \int_{-\infty}^{\infty}|\operatorname{det} B|^{2} . \\
& \cdot f\left(b_{1}\right) \ldots f\left(b_{n+1}\right) \cdot \frac{1}{2} \ldots \frac{1}{2} \cdot d b_{1}^{n} \ldots d b_{1}^{2} d b_{1}^{1} \ldots d b_{n+1}^{n} \ldots d b_{n+1}^{2} d b_{n+1}^{1}
\end{aligned}
$$


and

$$
\begin{aligned}
\Lambda_{W}(s, z)= & \int_{1-z}^{1-\frac{z(s-2)}{s}} \int_{-\infty}^{\infty} \ldots \int_{-\infty}^{\infty} \ldots \int_{1-z}^{1-\frac{z(s-2)}{s}} \int_{-\infty}^{\infty} \ldots \int_{-\infty}^{\infty} W\left(b_{1}, \ldots, b_{n}\right)^{2} . \\
& \cdot f\left(b_{1}\right) \ldots f\left(b_{n+1}\right) \cdot \frac{1}{2} \ldots \frac{1}{2} \cdot d b_{1}^{n} \ldots d b_{1}^{2} d b_{1}^{1} \ldots d b_{n+1}^{n} \ldots d b_{n+1}^{2} d b_{n+1}^{1}
\end{aligned}
$$

as well as

$$
\begin{aligned}
& g(s, z):=\frac{1}{2 z} \cdot \int_{1-z}^{1-\frac{z(s-2)}{s}} \int_{-\infty}^{\infty} \ldots \int_{-\infty}^{\infty} f\left(x^{1}, \ldots, x^{n}\right) \cdot d x^{n} \ldots d x^{2} d x^{1}, \\
& g_{2}(s, z):=\frac{1}{2 z} \cdot \int_{1-z}^{1-\frac{z(s-2)}{s}} \int_{-\infty}^{\infty} \ldots \int_{-\infty}^{\infty}\left(x^{1}\right)^{2} \cdot f\left(x^{1}, \ldots, x^{n}\right) \cdot d x^{n} \ldots d x^{2} d x^{1}, \\
& g_{3}(s, z):=\frac{1}{2 z} \cdot \int_{1-z} \int_{-\infty}^{\infty} \ldots \int_{-\infty}^{\infty}\left(x^{2}\right)^{2} \cdot f\left(x^{1}, \ldots, x^{n}\right) \cdot d x^{n} \ldots d x^{2} d x^{1}, \\
& g_{4}(s, z):=\frac{1}{2 z} \cdot \int_{1-z}^{\infty} \int_{-\infty}^{\infty} \ldots \int_{-\infty}^{\infty} x^{1} \cdot f\left(x^{1}, \ldots, x^{n}\right) \cdot d x^{n} \ldots d x^{2} d x^{1},
\end{aligned}
$$

so we obtain the following Lemma.

\section{Lemma 2.8 .}

$$
\Lambda_{B}(s, z)=(n+1) ! \cdot z^{n+1} \cdot g_{3}(s, z)^{n-1} \cdot\left[g(s, z) g_{2}(s, z)-g_{4}(s, z)^{2}\right]
$$

and for $z \leq 1$

$$
\Lambda_{W}(s, z) \leq \frac{1}{\left(\lambda_{n}\left(\Omega_{n}\right)\right)^{2} n !} \cdot \frac{1}{(1-z)^{2 n}} \cdot z^{n+1} \cdot g(s, z) \cdot g_{2}(s, z) \cdot g_{3}(s, z)^{n-1} .
$$

From the inequality of Cauchy-Schwarz we get a first estimation for $J_{C}^{z \leq 1}$.

Theorem 2.9. For any $q \in(0,1)$ holds

$$
\begin{aligned}
J_{C}^{z \leq 1} \leq\left(\begin{array}{c}
m \\
n+1
\end{array}\right) \cdot(n+1) \cdot \lambda_{n-1}\left(\omega_{n}\right) \cdot \int_{q}^{1} \int_{2}^{\infty} G(s, z)^{m-(n+1)} \cdot \frac{s^{n}}{z^{n+1}} \cdot \Lambda(s, z) \cdot d s d z+ \\
+\left(\begin{array}{c}
m \\
n+1
\end{array}\right) \cdot(n+1)^{\frac{3}{2}} \cdot \frac{\lambda_{n-1}\left(\omega_{n}\right)}{\lambda_{n}\left(\Omega_{n}\right)} \cdot \int_{0}^{q} \int_{2}^{\infty} G(s, z)^{m-(n+1)} \cdot \frac{s^{n}}{(1-z)^{n}} \cdot \\
\cdot g_{3}(s, z)^{n-1} \cdot \sqrt{g(s, z) g_{2}(s, z)\left[g(s, z) g_{2}(s, z)-g_{4}(s, z)^{2}\right]} \cdot d s d z .
\end{aligned}
$$


For the functions $\Phi=1-G$ and $g, g_{2}, g_{3}, g_{4}$ we know from [8] the following Lemma.

Lemma 2.10. It holds

$$
\Phi(s, z)=\left[g_{4}(s, z)-(1-z) g(s, z)\right] \cdot s+\frac{\Gamma\left(\kappa+1+\frac{n}{2}\right)}{\sqrt{\pi} \Gamma\left(\kappa+1+\frac{n-1}{2}\right)} \cdot \int_{1-\frac{z(s-2)}{s}}^{1}\left(1-\zeta^{2}\right)^{\frac{n-1}{2}+\kappa} d \zeta
$$

as well as

$$
\frac{\partial \Phi}{\partial z}(s, z)=\left[g(s, z)-g_{4}(s, z)\right] \cdot \frac{s}{z}
$$

In the case of our distribution family this time we obtain

$$
\begin{gathered}
g(s, z)=\frac{1}{2 z} \cdot \frac{\Gamma\left(\kappa+1+\frac{n}{2}\right)}{\sqrt{\pi} \Gamma\left(\kappa+1+\frac{n-1}{2}\right)} \cdot \int_{1-z}^{1-\frac{z(s-2)}{s}}\left(1-\zeta^{2}\right)^{\frac{n-1}{2}+\kappa} d \zeta, \\
g_{2}(s, z)=\frac{1}{2 z} \cdot \frac{\Gamma\left(\kappa+1+\frac{n}{2}\right)}{\sqrt{\pi} \Gamma\left(\kappa+1+\frac{n-1}{2}\right)} \cdot \int_{1-z}^{1-\frac{z(s-2)}{s}} \zeta^{2} \cdot\left(1-\zeta^{2}\right)^{\frac{n-1}{2}+\kappa} d \zeta, \\
g_{3}(s, z)=\frac{1}{2 z} \cdot \frac{\Gamma\left(\kappa+1+\frac{n}{2}\right)}{2 \sqrt{\pi} \Gamma\left(\kappa+1+\frac{n+1}{2}\right)} \cdot \int_{1-z}^{1-\frac{z(s-2)}{s}}\left(1-\zeta^{2}\right)^{\frac{n+1}{2}+\kappa} d \zeta, \\
g_{4}(s, z)=\frac{1}{2 z} \cdot \frac{\Gamma\left(\kappa+1+\frac{n}{2}\right)}{\sqrt{\pi} \Gamma\left(\kappa+1+\frac{n-1}{2}\right)} \cdot \int_{1-z}^{s} \zeta \cdot\left(1-\zeta^{2}\right)^{\frac{n-1}{2}+\kappa} d \zeta .
\end{gathered}
$$

Next we give some helpful Lemmas, which we will need for the main proof of the category C.

Lemma 2.11. For $s \in[2, \infty)$ there is a function $\alpha_{s}:(0,1] \rightarrow \mathbb{R}$ with

$$
\begin{array}{r}
g(s, z) g_{2}(s, z)-g_{4}(s, z)^{2}=\left[\frac{\Phi(s, z)}{s}\right]^{2} \cdot \frac{n+1+2 \kappa}{n+5+2 \kappa} \cdot \\
\cdot \frac{\left(1-\left(\frac{s-2}{s}\right)^{\frac{n+1}{2}+\kappa}\right)\left(1-\left(\frac{s-2}{s}\right)^{\frac{n+5}{2}+\kappa}\right)-4\left(\frac{n+1}{2}+\kappa\right)\left(\frac{n+5}{2}+\kappa\right) \frac{1}{s^{2}}\left(\frac{s-2}{s}\right)^{\frac{n+1}{2}+\kappa}}{\left(1-\left(\frac{s-2}{s}\right)^{\frac{n+3}{2}+\kappa}\right)^{2}} \cdot \\
\cdot\left(1+\alpha_{s}(z)\right)
\end{array}
$$

and $\alpha_{s}(z) \rightarrow 0$ for $z \rightarrow 0$.

This Lemma equates to the crucial Lemma 2.6 of the category A. It can be proven in the same manner as in the case of category A, even though the calculation becomes more complicated. The following technical Lemma can be seen as an appendix to the previous Lemma. 
Lemma 2.12. There is a function $\alpha:[2, \infty) \rightarrow \mathbb{R}$ with

$$
\begin{aligned}
\frac{\left(1-\left(\frac{s-2}{s}\right)^{\frac{n+1}{2}+\kappa}\right)\left(1-\left(\frac{s-2}{s}\right)^{\frac{n+5}{2}+\kappa}\right)-4\left(\frac{n+1}{2}+\kappa\right)\left(\frac{n+5}{2}+\kappa\right) \frac{1}{s^{2}}\left(\frac{s-2}{s}\right)^{\frac{n+1}{2}+\kappa}}{\left(1-\left(\frac{s-2}{s}\right)^{\frac{n+3}{2}+\kappa}\right)^{2}} \\
=\frac{1}{s^{2}} \cdot \frac{1}{3}\left(\frac{n+1}{2}+\kappa\right)\left(\frac{n+5}{2}+\kappa\right) \cdot(1+\alpha(s))
\end{aligned}
$$

and $\alpha(s) \rightarrow 0$ for $s \rightarrow \infty$.

Proof. First, we define

$$
\begin{aligned}
Z(s):=\left(1-\left(\frac{s-2}{s}\right)^{\frac{n+1}{2}+\kappa}\right) & \left(1-\left(\frac{s-2}{s}\right)^{\frac{n+5}{2}+\kappa}\right)- \\
& -4\left(\frac{n+1}{2}+\kappa\right)\left(\frac{n+5}{2}+\kappa\right) \frac{1}{s^{2}}\left(\frac{s-2}{s}\right)^{\frac{n+1}{2}+\kappa}
\end{aligned}
$$

and

$$
N(s):=\left(1-\left(\frac{s-2}{s}\right)^{\frac{n+3}{2}+\kappa}\right)^{2}
$$

Using the rule of l'Hospital we easily obtain

$$
\lim _{s \rightarrow \infty} \frac{1-\left(\frac{s-2}{s}\right)^{\frac{n+3}{2}+\kappa}}{\frac{1}{s}}=\lim _{s \rightarrow \infty} \frac{-\left(\frac{n+3}{2}+\kappa\right)\left(\frac{s-2}{s}\right)^{\frac{n+1}{2}+\kappa} \frac{2}{s^{2}}}{-\frac{1}{s^{2}}}=n+3+2 \kappa .
$$

So it holds

$$
N(s) \stackrel{s \rightarrow \infty}{\longrightarrow} \frac{1}{s^{2}} \cdot 4\left(\frac{n+3}{2}+\kappa\right)^{2}
$$

The numerator $Z(s)$ has an order of growth (for $s \rightarrow \infty$ ) like $\frac{1}{s^{4}}$. To show this, again, we use the rule of l'Hospital three times. In the following calculation we give the simplified terms of the derivations, which are already canceled by $\frac{1}{s^{2}}$ in each case. It is

$$
\lim _{s \rightarrow \infty} \frac{\left(1-\left(\frac{s-2}{s}\right)^{\frac{n+1}{2}+\kappa}\right)\left(1-\left(\frac{s-2}{s}\right)^{\frac{n+5}{2}+\kappa}\right)-4\left(\frac{n+1}{2}+\kappa\right)\left(\frac{n+5}{2}+\kappa\right) \frac{1}{s^{2}}\left(\frac{s-2}{s}\right)^{\frac{n+1}{2}+\kappa}}{\frac{1}{s^{4}}}
$$




$$
\begin{aligned}
& =\lim _{s \rightarrow \infty}\left\{-2\left(\frac{n+1}{2}+\kappa\right)\left(\frac{s-2}{s}\right)^{\frac{n-1}{2}+\kappa}\left(1-\left(\frac{s-2}{s}\right)^{\frac{n+5}{2}+\kappa}\right)-\right. \\
& -2\left(\frac{n+5}{2}+\kappa\right)\left(\frac{s-2}{s}\right)^{\frac{n+3}{2}+\kappa}\left(1-\left(\frac{s-2}{s}\right)^{\frac{n+1}{2}+\kappa}\right)+ \\
& +8\left(\frac{n+1}{2}+\kappa\right)\left(\frac{n+5}{2}+\kappa\right)\left(\frac{s-2}{s}\right)^{\frac{n+1}{2}+\kappa} \frac{1}{s}- \\
& \left.-8\left(\frac{n+1}{2}+\kappa\right)^{2}\left(\frac{n+5}{2}+\kappa\right)\left(\frac{s-2}{s}\right)^{\frac{n-1}{2}+\kappa} \frac{1}{s^{2}}\right\} /\left\{-\frac{4}{s^{3}}\right\} \\
& =\lim _{s \rightarrow \infty}\left\{-4\left(\frac{n+1}{2}+\kappa\right)\left(\frac{n-1}{2}+\kappa\right)\left(\frac{s-2}{s}\right)^{\frac{n-3}{2}+\kappa}\left(1-\left(\frac{s-2}{s}\right)^{\frac{n+5}{2}+\kappa}\right)-\right. \\
& -4\left(\frac{n+5}{2}+\kappa\right)\left(\frac{n+3}{2}+\kappa\right)\left(\frac{s-2}{s}\right)^{\frac{n+1}{2}+\kappa}\left(1-\left(\frac{s-2}{s}\right)^{\frac{n+1}{2}+\kappa}\right)- \\
& -8\left(\frac{n+1}{2}+\kappa\right)\left(\frac{n+5}{2}+\kappa\right)\left(\frac{s-2}{s}\right)^{\frac{n+1}{2}+\kappa}\left(1-\left(\frac{s-2}{s}\right)^{\frac{n+1}{2}+\kappa}\right)+ \\
& +32\left(\frac{n+1}{2}+\kappa\right)^{2}\left(\frac{n+5}{2}+\kappa\right)\left(\frac{s-2}{s}\right)^{\frac{n-1}{2}+\kappa} \frac{1}{s}- \\
& \left.-16\left(\frac{n+1}{2}+\kappa\right)^{2}\left(\frac{n+5}{2}+\kappa\right)\left(\frac{n-1}{2}+\kappa\right)\left(\frac{s-2}{s}\right)^{\frac{n-3}{2}+\kappa} \frac{1}{s^{2}}\right\} /\left\{\frac{12}{s^{2}}\right\} \\
& =\lim _{s \rightarrow \infty}\{ \\
& -8\left(\frac{n+1}{2}+\kappa\right)\left(\frac{n-1}{2}+\kappa\right)\left(\frac{n-3}{2}+\kappa\right)\left(\frac{s-2}{s}\right)^{\frac{n-5}{2}+\kappa}\left(1-\left(\frac{s-2}{s}\right)^{\frac{n+5}{2}+\kappa}\right)- \\
& -8\left(\frac{n+5}{2}+\kappa\right)\left(\frac{n+3}{2}+\kappa\right)\left(\frac{n+1}{2}+\kappa\right)\left(\frac{s-2}{s}\right)^{\frac{n-1}{2}+\kappa}\left(1-\left(\frac{s-2}{s}\right)^{\frac{n+1}{2}+\kappa}\right)- \\
& -48\left(\frac{n+1}{2}+\kappa\right)^{2}\left(\frac{n+5}{2}+\kappa\right)\left(\frac{s-2}{s}\right)^{\frac{n-1}{2}+\kappa}\left(1-\left(\frac{s-2}{s}\right)^{\frac{n+1}{2}+\kappa}\right)+ \\
& +96\left(\frac{n+1}{2}+\kappa\right)^{2}\left(\frac{n+5}{2}+\kappa\right)\left(\frac{n-1}{2}+\kappa\right)\left(\frac{s-2}{s}\right)^{\frac{n-3}{2}+\kappa} \frac{1}{s}- \\
& \left.-32\left(\frac{n+1}{2}+\kappa\right)^{2}\left(\frac{n+5}{2}+\kappa\right)\left(\frac{n-1}{2}+\kappa\right)\left(\frac{n-3}{2}+\kappa\right)\left(\frac{s-2}{s}\right)^{\frac{n-5}{2}+\kappa} \frac{1}{s^{2}}\right\} / \\
& \left\{-\frac{24}{s}\right\}
\end{aligned}
$$




$$
=\frac{4}{3}\left(\frac{n+1}{2}+\kappa\right)\left(\frac{n+5}{2}+\kappa\right)\left(\frac{n+3}{2}+\kappa\right)^{2} .
$$

So we have

$$
Z(s) \stackrel{s \rightarrow \infty}{\longrightarrow} \frac{1}{s^{4}} \cdot \frac{4}{3}\left(\frac{n+1}{2}+\kappa\right)\left(\frac{n+5}{2}+\kappa\right)\left(\frac{n+3}{2}+\kappa\right)^{2} .
$$

For the whole quotient we finally obtain

$$
\frac{Z(s)}{N(s)} \stackrel{s \rightarrow \infty}{\longrightarrow} \frac{1}{s^{2}} \cdot \frac{1}{3}\left(\frac{n+1}{2}+\kappa\right)\left(\frac{n+5}{2}+\kappa\right)
$$

and the claim is obvious.

Lemma 2.13. For $z \in[0,1]$ there is a function $\alpha_{z}:[2, \infty) \rightarrow \mathbb{R}$ with

$$
\Phi(s, z)=\frac{\Gamma\left(\kappa+1+\frac{n}{2}\right)}{\sqrt{\pi} \Gamma\left(\kappa+1+\frac{n-1}{2}\right)} \cdot \int_{1-z}^{1}\left(1-\zeta^{2}\right)^{\frac{n-1}{2}+\kappa} d \zeta \cdot\left(1+\alpha_{z}(s)\right)
$$

and $\alpha_{z}(s) \rightarrow 0$ for $s \rightarrow \infty$.

Proof. From Lemma 2.10 we know

$$
\Phi(s, z)=\left[g_{4}(s, z)-(1-z) g(s, z)\right] \cdot s+\frac{\Gamma\left(\kappa+1+\frac{n}{2}\right)}{\sqrt{\pi} \Gamma\left(\kappa+1+\frac{n-1}{2}\right)} \cdot \int_{1-\frac{z(s-2)}{s}}^{1}\left(1-\zeta^{2}\right)^{\frac{n-1}{2}+\kappa} d \zeta .
$$

If we can show that

$$
\left[g_{4}(s, z)-(1-z) g(s, z)\right] \cdot s \stackrel{s \rightarrow \infty}{\longrightarrow} 0
$$

this would prove our claim. For that purpose we use the rule of l'Hospital, which yields

$$
\begin{gathered}
\lim _{s \rightarrow \infty} \frac{\int_{1-z}^{1-\frac{z(s-2)}{s}} \zeta\left(1-\zeta^{2}\right)^{\frac{n-1}{2}+\kappa} d \zeta-(1-z) \int_{1-z}^{1-\frac{z(s-2)}{s}}\left(1-\zeta^{2}\right)^{\frac{n-1}{2}+\kappa} d \zeta}{\frac{1}{s}=\lim _{s \rightarrow \infty}} \\
\frac{-\frac{2 z}{s^{2}}\left(1-\frac{z(s-2)}{s}\right)\left(1-\left(1-\frac{z(s-2)}{s}\right)^{2}\right)^{\frac{n-1}{2}+\kappa}+\frac{2 z}{s^{2}}(1-z)\left(1-\left(1-\frac{z(s-2)}{s}\right)^{2}\right)^{\frac{n-1}{2}+\kappa}}{-\frac{1}{s^{2}}}=0 .
\end{gathered}
$$


Lemma 2.14. For $z \in[0,1]$ there is a function $\alpha_{z}:[2, \infty) \rightarrow \mathbb{R}$ with

$$
g(s, z)=\frac{1}{s} \cdot \frac{\Gamma\left(\kappa+1+\frac{n}{2}\right)}{\sqrt{\pi} \Gamma\left(\kappa+1+\frac{n-1}{2}\right)} \cdot\left(1-(1-z)^{2}\right)^{\frac{n-1}{2}+\kappa} \cdot\left(1+\alpha_{z}(s)\right)
$$

and $\alpha_{z}(s) \rightarrow 0$ for $s \rightarrow \infty$.

Proof. The rule of l'Hospital yields

$$
\begin{aligned}
& \lim _{s \rightarrow \infty} \frac{1-\frac{z(s-2)}{\int_{1-z}^{s}}\left(1-\zeta^{2}\right)^{\frac{n-1}{2}+\kappa} d \zeta}{\frac{1}{s}}=\lim _{s \rightarrow \infty} \frac{-2 \frac{z}{s^{2}}\left(1-\left(1-\frac{z(s-2)}{s}\right)^{2}\right)^{\frac{n-1}{2}+\kappa}}{-\frac{1}{s^{2}}} \\
& =2 z\left(1-(1-z)^{2}\right)^{\frac{n-1}{2}+\kappa}
\end{aligned}
$$

and the claim is obvious.

Analogously we obtain the following fact.

Lemma 2.15. For $z \in[0,1]$ there is a function $\alpha_{z}:[2, \infty) \rightarrow \mathbb{R}$ with

$$
g_{3}(s, z)=\frac{1}{s} \cdot \frac{\Gamma\left(\kappa+1+\frac{n}{2}\right)}{2 \sqrt{\pi} \Gamma\left(\kappa+1+\frac{n+1}{2}\right)} \cdot\left(1-(1-z)^{2}\right)^{\frac{n+1}{2}+\kappa} \cdot\left(1+\alpha_{z}(s)\right)
$$

and $\alpha_{z}(s) \rightarrow 0$ for $s \rightarrow \infty$.

More complicated to prove, but very essential is the last Lemma in this series.

Lemma 2.16. For $z \in[0,1]$ there is a function $\alpha_{z}:[2, \infty) \rightarrow \mathbb{R}$ with

$$
\begin{aligned}
g(s, z) g_{2}(s, z) & -g_{4}(s, z)^{2} \\
= & \frac{1}{s^{4}} \cdot \frac{1}{3} \cdot\left[\frac{\Gamma\left(\kappa+1+\frac{n}{2}\right)}{\sqrt{\pi} \Gamma\left(\kappa+1+\frac{n-1}{2}\right)}\right]^{2} \cdot\left[z\left(1-(1-z)^{2}\right)^{\frac{n-1}{2}+\kappa}\right]^{2} \cdot\left(1+\alpha_{z}(s)\right)
\end{aligned}
$$

and $\alpha_{z}(s) \rightarrow 0$ for $s \rightarrow \infty$.

Proof. First, we recall

$$
\begin{aligned}
& g(s, z) g_{2}(s, z)-g_{4}(s, z)^{2}=\left[\frac{1}{2 z} \cdot \frac{\Gamma\left(\kappa+1+\frac{n}{2}\right)}{\sqrt{\pi} \Gamma\left(\kappa+1+\frac{n-1}{2}\right)}\right]^{2} \cdot \\
& {\left[\int_{1-z}^{1-\frac{z(s-2)}{s}}\left(1-\zeta^{2}\right)^{\frac{n-1}{2}+\kappa} d \zeta \int_{1-z}^{1-\frac{z(s-2)}{s}} \zeta^{2}\left(1-\zeta^{2}\right)^{\frac{n-1}{2}+\kappa} d \zeta-\left[\int_{1-z}^{1-\frac{z(s-2)}{s}} \zeta\left(1-\zeta^{2}\right)^{\frac{n-1}{2}+\kappa} d \zeta\right]^{2}\right]}
\end{aligned}
$$


where the first factor is only a constant in $s$. Now, we apply the rule of l'Hospital four times and reduce the fraction by the term $\frac{1}{s^{2}}$ after each step.

$$
\begin{aligned}
& \lim _{s \rightarrow \infty}\left\{\int_{1-z}^{1-\frac{z(s-2)}{s}}\left(1-\zeta^{2}\right)^{\frac{n-1}{2}+\kappa} d \zeta \int_{1-z}^{1-\frac{z(s-2)}{s}} \zeta^{2}\left(1-\zeta^{2}\right)^{\frac{n-1}{2}+\kappa} d \zeta-\right. \\
& \left.-\left[\int_{1-z}^{1-\frac{z(s-2)}{s}} \zeta\left(1-\zeta^{2}\right)^{\frac{n-1}{2}+\kappa} d \zeta\right]^{2}\right\} /\left\{\frac{1}{s^{4}}\right\} \\
& =\lim _{s \rightarrow \infty}\left\{2 z\left(1-\left(1-\frac{z(s-2)}{s}\right)^{2}\right)^{\frac{n-1}{2}+\kappa}\right\} \text {. } \\
& \lim _{s \rightarrow \infty}\left\{2\left(1-\frac{z(s-2)}{s}\right) \int_{1-z}^{1-\frac{z(s-2)}{s}} \zeta\left(1-\zeta^{2}\right)^{\frac{n-1}{2}+\kappa} d \zeta-\right. \\
& \left.-\left(1-\frac{z(s-2)}{s}\right)^{2} \int_{1-z}^{1-\frac{z(s-2)}{s}}\left(1-\zeta^{2}\right)^{\frac{n-1}{2}+\kappa} d \zeta-\int_{1-z}^{1-\frac{z(s-2)}{s}} \zeta^{2}\left(1-\zeta^{2}\right)^{\frac{n-1}{2}+\kappa} d \zeta\right\} /\left\{-\frac{4}{s^{3}}\right\} \\
& =\left\{8 z^{2}\left(1-(1-z)^{2}\right)^{\frac{n-1}{2}+\kappa}\right\} \text {. } \\
& \cdot \lim _{s \rightarrow \infty}\left\{\left(1-\frac{z(s-2)}{s}\right) \int_{1-z}^{1-\frac{z(s-2)}{s}}\left(1-\zeta^{2}\right)^{\frac{n-1}{2}+\kappa} d \zeta-\int_{1-z}^{1-\frac{z(s-2)}{s}} \zeta\left(1-\zeta^{2}\right)^{\frac{n-1}{2}+\kappa} d \zeta\right\} /\left\{\frac{12}{s^{2}}\right\} \\
& =\left\{16 z^{3}\left(1-(1-z)^{2}\right)^{\frac{n-1}{2}+\kappa}\right\} \cdot \lim _{s \rightarrow \infty}\left\{-\int_{1-z}^{1-\frac{z(s-2)}{s}}\left(1-\zeta^{2}\right)^{\frac{n-1}{2}+\kappa} d \zeta\right\} /\left\{-\frac{24}{s}\right\} \\
& =\left\{16 z^{3}\left(1-(1-z)^{2}\right)^{\frac{n-1}{2}+\kappa}\right\} \cdot \lim _{s \rightarrow \infty}\left\{2 z \frac{1}{s^{2}}\left(1-\left(1-\frac{z(s-2)}{s}\right)^{2}\right)^{\frac{n-1}{2}+\kappa}\right\} /\left\{\frac{24}{s^{2}}\right\} \\
& =\frac{4}{3} z^{4}\left[\left(1-(1-z)^{2}\right)^{\frac{n-1}{2}+\kappa}\right]^{2} \text {. }
\end{aligned}
$$

Taking into regard the constant factor from the beginning the claim results.

Now, we can prove our main result for the category C. 
Theorem 2.17. For $n \geq 3$ there is a function $\varepsilon_{\kappa}(m, n)$, which depends on the distribution parameter $\kappa$, with $\varepsilon_{\kappa}(m, n) \rightarrow 0$ for $m \rightarrow \infty, n$ constant and

$$
\begin{array}{r}
J_{C}^{z \leq 1}=\left(\begin{array}{c}
m \\
n+1
\end{array}\right) \cdot(n+1) \cdot \lambda_{n-1}\left(\omega_{n}\right) \cdot \int_{0}^{1} \int_{2}^{\infty} G(s, z)^{m-(n+1)} \cdot \frac{s^{n}}{z^{n+1}} \cdot \Lambda(s, z) \cdot d s d z \\
\leq C_{C}(\kappa, n) \cdot\left(1+\varepsilon_{\kappa}(m, n)\right)
\end{array}
$$

where $C_{C}(\kappa, n)$ is a constant depending only on the dimension $n$ and the distribution parameter $\kappa$.

Proof. For $q \in(0,1)$ and $t \in(2, \infty)$ first we define

$$
J_{C}^{1}:=\left(\begin{array}{c}
m \\
n+1
\end{array}\right) \cdot(n+1) \cdot \lambda_{n-1}\left(\omega_{n}\right) \cdot \int_{q}^{1} \int_{2}^{\infty} G(s, z)^{m-(n+1)} \cdot \frac{s^{n}}{z^{n+1}} \cdot \Lambda(s, z) \cdot d s d z
$$

and

$$
J_{C}^{2}:=\left(\begin{array}{c}
m \\
n+1
\end{array}\right) \cdot(n+1) \cdot \lambda_{n-1}\left(\omega_{n}\right) \cdot \int_{0}^{q} \int_{2}^{t} G(s, z)^{m-(n+1)} \cdot \frac{s^{n}}{z^{n+1}} \cdot \Lambda(s, z) \cdot d s d z
$$

as well as

$$
J_{C}^{3}:=\left(\begin{array}{c}
m \\
n+1
\end{array}\right) \cdot(n+1) \cdot \lambda_{n-1}\left(\omega_{n}\right) \cdot \int_{0}^{q} \int_{t}^{\infty} G(s, z)^{m-(n+1)} \cdot \frac{s^{n}}{z^{n+1}} \cdot \Lambda(s, z) \cdot d s d z
$$

so that obviously $J_{C}^{z \leq 1}=J_{C}^{1}+J_{C}^{2}+J_{C}^{3}$. The term $J_{C}^{1}$ is asymptotically not relevant because we have

$$
G(s, z) \leq G(2, q)<1
$$

in this case. Because of

$$
\left(\begin{array}{c}
m \\
n+1
\end{array}\right)=\frac{m !}{(m-n-1) !(n+1) !} \leq m^{n+1}
$$

the whole integral $J_{C}^{1}$ therefore can be estimated by

$$
J_{C}^{1} \leq C_{1}(\kappa, n) \cdot m^{n+1} \cdot G(2, q)^{m-(n+1)}
$$

with a constant $C_{1}(\kappa, n)$ depending only on the dimension $n$ and the distribution parameter $\kappa$. Since $G(2, q)<1$, this tends to 0 for $m \rightarrow \infty$. Now, we care about $J_{C}^{2}$. For $q \leq 1$ it follows from Theorem 2.9 and the obvious fact $g_{2}(s, z) \leq g(s, z)$ that

$$
\begin{aligned}
& J_{C}^{2} \leq\left(\begin{array}{c}
m \\
n+1
\end{array}\right) \cdot(n+1)^{\frac{3}{2}} \cdot \frac{\lambda_{n-1}\left(\omega_{n}\right)}{\lambda_{n}\left(\Omega_{n}\right)} \cdot \int_{0}^{q} \int_{2}^{t} G(s, z)^{m-(n+1)} \cdot \frac{s^{n}}{(1-z)^{n}} \cdot \\
& \frac{g(s, z)-g_{4}(s, z)}{z} \cdot g_{3}(s, z)^{n-1} \cdot z \cdot \frac{\sqrt{g(s, z) g_{2}(s, z)\left[g(s, z) g_{2}(s, z)-g_{4}(s, z)^{2}\right]}}{g(s, z)-g_{4}(s, z)} \cdot d s d z
\end{aligned}
$$




$$
\begin{gathered}
\leq\left(\begin{array}{c}
m \\
n+1
\end{array}\right) \cdot \frac{(n+1)^{\frac{3}{2}}}{(1-q)^{n}} \cdot \frac{\lambda_{n-1}\left(\omega_{n}\right)}{\lambda_{n}\left(\Omega_{n}\right)} \cdot \int_{0}^{q} \int_{2}^{t} G(s, z)^{m-(n+1)} \cdot s^{n} . \\
\frac{g(s, z)-g_{4}(s, z)}{z} \cdot g_{3}(s, z)^{n-1} \cdot g(s, z) \cdot z \cdot \frac{\sqrt{g(s, z) g_{2}(s, z)-g_{4}(s, z)^{2}}}{g(s, z)-g_{4}(s, z)} \cdot d s d z .
\end{gathered}
$$

From [8] and Lemma 2.11 we know that for any $\delta>0$ we can choose a $q \in(0,1)$ depending on the dimension $n$ and the distribution parameter $\kappa$ as well as $t$ small enough so that for any $z \in[0, q]$ holds

1. $g_{3}(s, z) \leq \frac{\Phi(s, z)}{s} \cdot(1+\delta)$

2. $g(s, z) \leq \frac{\Phi(s, z)^{\frac{n-1+2 \kappa}{n+1+2 \kappa}}}{s} \cdot\left[\kappa+1+\frac{n-1}{2}\right]^{\frac{n-1+2 \kappa}{n+1+2 \kappa}} \cdot\left[\frac{\Gamma\left(\kappa+1+\frac{n}{2}\right)}{\sqrt{\pi} \Gamma\left(\kappa+1+\frac{n-1}{2}\right)}\right]^{\frac{2}{n+1+2 \kappa}}$

3. $\frac{1}{(1-q)^{n}} \leq 1+\delta$

4. $\sqrt{g(s, z) g_{2}(s, z)-g_{4}(s, z)^{2}} \leq \frac{\Phi(s, z)}{s} \cdot \sqrt{\frac{n+1+2 \kappa}{n+5+2 \kappa}}$.

$\sqrt{\frac{\left(1-\left(\frac{s-2}{s}\right)^{\frac{n+1}{2}+\kappa}\right)\left(1-\left(\frac{s-2}{s}\right)^{\frac{n+5}{2}+\kappa}\right)-4\left(\frac{n+1}{2}+\kappa\right)\left(\frac{n+5}{2}+\kappa\right) \frac{1}{s^{2}}\left(\frac{s-2}{s}\right)^{\frac{n+1}{2}+\kappa}}{\left(1-\left(\frac{s-2}{s}\right)^{\frac{n+3}{2}+\kappa}\right)^{2}}}$.

$\cdot(1+\delta)$

5. $g(s, z)-g_{4}(s, z) \geq \frac{\Phi(s, z)}{s} \cdot\left(\frac{n+1}{2}+\kappa\right) \cdot(1+\delta)^{-1}$,

6. $z \leq \Phi(s, z)^{\frac{2}{n+1+2 \kappa}} \cdot\left[\frac{\left(\frac{n+1}{2}+\kappa\right)\left(\frac{n+3}{2}+\kappa\right) \frac{\sqrt{\pi} \Gamma\left(\kappa+1+\frac{n-1}{2}\right)}{\Gamma\left(\kappa+1+\frac{n}{2}\right)}}{2^{\frac{n-3}{2}+\kappa} s\left(1-\left(\frac{s-2}{s}\right)^{\frac{n+3}{2}+\kappa}\right)}\right]^{\frac{2}{n+1+2 \kappa}} \cdot(1+\delta)$. 
So for a suitable $q$ we get

$$
\begin{aligned}
& J_{C}^{2} \leq\left(\begin{array}{c}
m \\
n+1
\end{array}\right) \cdot(n+1)^{\frac{3}{2}} \cdot \frac{\lambda_{n-1}\left(\omega_{n}\right)}{\lambda_{n}\left(\Omega_{n}\right)} \cdot \sqrt{\frac{n+1+2 \kappa}{n+5+2 \kappa}} \\
& \cdot \frac{\left(\frac{n+3}{2}+\kappa\right)^{\frac{2}{n+1+2 \kappa}}}{2^{\frac{n-3+2 \kappa}{n+1+2 \kappa}}} \cdot \int_{2}^{t} \int_{0}^{q}\left[\frac{\frac{1}{s}}{1-\left(\frac{s-2}{s}\right)^{\frac{n+3}{2}+\kappa}}\right]^{\frac{2}{n+1+2 \kappa}} \text {. } \\
& \sqrt{\frac{\left(1-\left(\frac{s-2}{s}\right)^{\frac{n+1}{2}+\kappa}\right)\left(1-\left(\frac{s-2}{s}\right)^{\frac{n+5}{2}+\kappa}\right)-4\left(\frac{n+1}{2}+\kappa\right)\left(\frac{n+5}{2}+\kappa\right) \frac{1}{s^{2}}\left(\frac{s-2}{s}\right)^{\frac{n+1}{2}+\kappa}}{\left(1-\left(\frac{s-2}{s}\right)^{\frac{n+3}{2}+\kappa}\right)^{2}}} \text {. } \\
& \cdot[1-\Phi(s, z)]^{m-(n+1)} \cdot \Phi(s, z)^{n-1} \cdot \frac{\Phi(s, z)^{\frac{n-1+2 \kappa}{n+1+2 \kappa}}}{s} \cdot \Phi(s, z)^{\frac{2}{n+1+2 \kappa}} . \\
& \cdot \frac{\Phi(s, z)}{s} \cdot\left[\frac{\Phi(s, z)}{s}\right]^{-1} \cdot \frac{\left[g(s, z)-g_{4}(s, z)\right] s}{z} \cdot d z d s \cdot(1+\delta)^{n+4} \\
& \leq\left(\begin{array}{c}
m \\
n+1
\end{array}\right) \cdot(n+1)^{\frac{3}{2}} \cdot \frac{\lambda_{n-1}\left(\omega_{n}\right)}{\lambda_{n}\left(\Omega_{n}\right)} \cdot \sqrt{\frac{n+1+2 \kappa}{n+5+2 \kappa}} \cdot \frac{\left(\frac{n+3}{2}+\kappa\right)^{\frac{2}{n+1+2 \kappa}}}{2^{\frac{n-3+2 \kappa}{n+1+2 \kappa}}} . \\
& \cdot \int_{2}^{t} \sqrt{\frac{\left(1-\left(\frac{s-2}{s}\right)^{\frac{n+1}{2}+\kappa}\right)\left(1-\left(\frac{s-2}{s}\right)^{\frac{n+5}{2}+\kappa}\right)-4\left(\frac{n+1}{2}+\kappa\right)\left(\frac{n+5}{2}+\kappa\right) \frac{1}{s^{2}}\left(\frac{s-2}{s}\right)^{\frac{n+1}{2}+\kappa}}{\left(1-\left(\frac{s-2}{s}\right)^{\frac{n+3}{2}+\kappa}\right)^{2}}} . \\
& \cdot \frac{1}{s} \cdot\left[\frac{\frac{1}{s}}{1-\left(\frac{s-2}{s}\right)^{\frac{n+3}{2}+\kappa}}\right]^{\frac{2}{n+1+2 \kappa}} d s \cdot \int_{0}^{1}(1-\Phi)^{m-(n+1)} \cdot \Phi^{(n+1)-1} d \Phi \cdot(1+\delta)^{n+4} .
\end{aligned}
$$

Analogously to the analysis of category A we have performed here the transformation $(s, \Phi) \mapsto(s, \Phi(s, z))$ with determinant of the Jacobian

$$
\operatorname{det}\left(\begin{array}{cc}
1 & 0 \\
\frac{\partial \Phi}{\partial s}(s, z) & \frac{\partial \Phi}{\partial z}(s, z)
\end{array}\right)=\frac{\partial \Phi}{\partial z}(s, z) \stackrel{\text { Lemma } 2.10}{=} \frac{\left[g(s, z)-g_{4}(s, z)\right] s}{z} .
$$

From the appendix of [4] we know

$$
\left(\begin{array}{c}
m \\
n+1
\end{array}\right) \cdot \int_{0}^{1}(1-\Phi)^{m-(n+1)} \cdot \Phi^{(n+1)-1} d \Phi=\frac{1}{n+1} .
$$

Taking into regard

$$
\left[\frac{\frac{1}{s}}{1-\left(\frac{s-2}{s}\right)^{\frac{n+3}{2}+\kappa}}\right]^{\frac{2}{n+1+2 \kappa}} \leq\left[\frac{\frac{1}{s}}{1-\frac{s-2}{s}}\right]^{\frac{2}{n+1+2 \kappa}}=\frac{1}{2^{\frac{2}{n+1+2 \kappa}}}
$$


as well as Lemma 2.12 we obtain

$$
\begin{gathered}
\int_{2}^{t} \sqrt{\frac{\left(1-\left(\frac{s-2}{s}\right)^{\frac{n+1}{2}+\kappa}\right)\left(1-\left(\frac{s-2}{s}\right)^{\frac{n+5}{2}+\kappa}\right)-4\left(\frac{n+1}{2}+\kappa\right)\left(\frac{n+5}{2}+\kappa\right) \frac{1}{s^{2}}\left(\frac{s-2}{s}\right)^{\frac{n+1}{2}+\kappa}}{\left(1-\left(\frac{s-2}{s}\right)^{\frac{n+3}{2}+\kappa}\right)^{2}}} . \\
\cdot \frac{1}{s} \cdot\left[\frac{\frac{1}{s}}{1-\left(\frac{s-2}{s}\right)^{\frac{n+3}{2}+\kappa}}\right]^{\frac{2}{n+1+2 \kappa}} d s \leq C_{S}(\kappa, n)
\end{gathered}
$$

where $C_{S}(\kappa, n)$ is a constant depending only on the dimension $n$ and the distribution parameter $\kappa$. Notice that $C_{S}(\kappa, n)$ depends not on $t$. So we finally get

$$
J_{C}^{2} \leq C_{2}(\kappa, n) \cdot(1+\delta)^{n+4}
$$

with a constant $C_{2}(\kappa, n)$. For $J_{C}^{3}$ similarly to $J_{C}^{2}$ we have

$$
\begin{aligned}
J_{C}^{3} \leq\left(\begin{array}{c}
m \\
n+1
\end{array}\right) \cdot(n+1)^{\frac{3}{2}} \cdot \frac{\lambda_{n-1}\left(\omega_{n}\right)}{\lambda_{n}\left(\Omega_{n}\right)} \cdot \int_{0}^{q} \int_{t}^{\infty} G(s, z)^{m-(n+1)} \cdot \frac{s^{n}}{(1-z)^{n+1}} \cdot \\
\cdot g_{3}(s, z)^{n-1} \cdot \sqrt{g(s, z) g_{2}(s, z)\left[g(s, z) g_{2}(s, z)-g_{4}(s, z)^{2}\right]} \cdot(1-z) \cdot d s d z \\
\leq\left(\begin{array}{c}
m \\
n+1
\end{array}\right) \cdot \frac{(n+1)^{\frac{3}{2}}}{(1-q)^{n+1}} \cdot \frac{\lambda_{n-1}\left(\omega_{n}\right)}{\lambda_{n}\left(\Omega_{n}\right)} \cdot \int_{0}^{q} \int_{t}^{\infty}[1-\Phi(s, z)]^{m-(n+1)} \cdot s^{n} \cdot \\
\cdot g_{3}(s, z)^{n-1} \cdot g(s, z) \cdot \sqrt{g(s, z) g_{2}(s, z)-g_{4}(s, z)^{2}} \cdot(1-z) \cdot d s d z .
\end{aligned}
$$

From our auxiliary Lemmas, which we have proven above, we know that for any $\delta>0$ we can choose $t \in(2, \infty)$ big enough (depending on the dimension $n$ and the distribution parameter $\kappa)$ so that for any $s \in[t, \infty)$ holds

1. $\Phi(s, z) \geq \frac{\Gamma\left(\kappa+1+\frac{n}{2}\right)}{\sqrt{\pi} \Gamma\left(\kappa+1+\frac{n-1}{2}\right)} \cdot \int_{1-z}^{1}\left(1-\zeta^{2}\right)^{\frac{n-1}{2}+\kappa} d \zeta \cdot(1+\delta)^{-1}$,

2. $g_{3}(s, z) \leq \frac{1}{s} \cdot \frac{\Gamma\left(\kappa+1+\frac{n}{2}\right)}{2 \sqrt{\pi} \Gamma\left(\kappa+1+\frac{n+1}{2}\right)} \cdot\left(1-(1-z)^{2}\right)^{\frac{n+1}{2}+\kappa} \cdot(1+\delta)$,

3. $g(s, z) \leq \frac{1}{s} \cdot \frac{\Gamma\left(\kappa+1+\frac{n}{2}\right)}{\sqrt{\pi} \Gamma\left(\kappa+1+\frac{n-1}{2}\right)} \cdot\left(1-(1-z)^{2}\right)^{\frac{n-1}{2}+\kappa} \cdot(1+\delta)$,

4. $\sqrt{g(s, z) g_{2}(s, z)-g_{4}(s, z)^{2}} \leq \frac{1}{s^{2}} \cdot \frac{1}{\sqrt{3}} \cdot\left[\frac{\Gamma\left(\kappa+1+\frac{n}{2}\right)}{\sqrt{\pi} \Gamma\left(\kappa+1+\frac{n-1}{2}\right)}\right]$.

$$
\cdot\left[z\left(1-(1-z)^{2}\right)^{\frac{n-1}{2}+\kappa}\right] \cdot(1+\delta) \text {. }
$$


So for a suitable $t$ we get

$$
\begin{aligned}
& J_{C}^{3} \leq\left(\begin{array}{c}
m \\
n+1
\end{array}\right) \cdot \frac{(n+1)^{\frac{3}{2}}}{(1-q)^{n+1}} \cdot \frac{\lambda_{n-1}\left(\omega_{n}\right)}{\lambda_{n}\left(\Omega_{n}\right)} \cdot\left[\frac{\Gamma\left(\kappa+1+\frac{n}{2}\right)}{\sqrt{\pi} \Gamma\left(\kappa+1+\frac{n-1}{2}\right)}\right]^{2} \cdot \\
& \cdot\left[\frac{\Gamma\left(\kappa+1+\frac{n}{2}\right)}{2 \sqrt{\pi} \Gamma\left(\kappa+1+\frac{n+1}{2}\right)}\right]^{n-1} \cdot \frac{1}{\sqrt{3}} \cdot \int_{t}^{\infty} \frac{1}{s^{2}} d s \cdot \\
& \\
& \cdot \int_{0}^{q}\left[1-\frac{\Gamma\left(\kappa+1+\frac{n}{2}\right)}{\sqrt{\pi} \Gamma\left(\kappa+1+\frac{n-1}{2}\right)} \cdot \int_{1-z}^{1}\left(1-\zeta^{2}\right)^{\frac{n-1}{2}+\kappa} d \zeta \cdot(1+\delta)^{-1}\right]^{m-(n+1)} \cdot \\
& \cdot\left[\left(1-(1-z)^{2}\right)^{\frac{n+1}{2}+\kappa}\right]^{n-1} \cdot\left[\left(1-(1-z)^{2}\right)^{\frac{n-1}{2}+\kappa}\right]^{2} \cdot z \cdot(1-z) d z \cdot(1+\delta)^{n+1} .
\end{aligned}
$$

Because of

$$
\lim _{z \rightarrow 0} \frac{\int_{1-z}^{1}\left(1-\zeta^{2}\right)^{\frac{n-1}{2}+\kappa} d \zeta}{\left(1-(1-z)^{2}\right)^{\frac{n+1}{2}+\kappa}}=\lim _{z \rightarrow 0} \frac{\left(1-(1-z)^{2}\right)^{\frac{n-1}{2}+\kappa}}{\left(\frac{n+1}{2}+\kappa\right) 2(1-z)\left(1-(1-z)^{2}\right)^{\frac{n-1}{2}+\kappa}}=\frac{1}{n+1+2 \kappa}
$$

and

$$
z\left(1-(1-z)^{2}\right)^{\frac{n-1}{2}+\kappa}=\frac{\left(1-(1-z)^{2}\right)^{\frac{n+1}{2}+\kappa}}{2-z} \stackrel{z \leq 1}{\leq}\left(1-(1-z)^{2}\right)^{\frac{n+1}{2}+\kappa}
$$

we obtain

$$
\begin{aligned}
J_{C}^{3} \leq\left(\begin{array}{c}
m \\
n+1
\end{array}\right) \cdot(n+1)^{\frac{3}{2}} \cdot \frac{\lambda_{n-1}\left(\omega_{n}\right)}{\lambda_{n}\left(\Omega_{n}\right)} \cdot\left[\frac{\Gamma\left(\kappa+1+\frac{n}{2}\right)}{\sqrt{\pi} \Gamma\left(\kappa+1+\frac{n-1}{2}\right)}\right]^{2} \cdot \\
\cdot\left[\frac{\Gamma\left(\kappa+1+\frac{n}{2}\right)}{2 \sqrt{\pi} \Gamma\left(\kappa+1+\frac{n+1}{2}\right)}\right]^{n-1} \cdot \frac{1}{\sqrt{3}} \cdot \int_{t}^{\infty} \frac{1}{s^{2}} d s \cdot \\
\quad \int_{0}^{q}\left[1-\frac{\Gamma\left(\kappa+1+\frac{n}{2}\right)}{\sqrt{\pi} \Gamma\left(\kappa+1+\frac{n-1}{2}\right)} \cdot \frac{\left(1-(1-z)^{2}\right)^{\frac{n+1}{2}+\kappa}}{n+1+2 \kappa} \cdot(1+\delta)^{-2}\right]^{m-(n+1)} \cdot \\
\cdot\left[\left(1-(1-z)^{2}\right)^{\frac{n+1}{2}+\kappa}\right]^{n} \cdot\left(1-(1-z)^{2}\right)^{\frac{n-1}{2}+\kappa} \cdot(1-z) d z \cdot(1+\delta)^{n+2},
\end{aligned}
$$

if we additionally choose $q \in(0,1)$ (depending on the dimension $n$ and the distribution parameter $\kappa$ ) small enough. Substituting

$$
\Phi=\frac{\Gamma\left(\kappa+1+\frac{n}{2}\right)}{\sqrt{\pi} \Gamma\left(\kappa+1+\frac{n-1}{2}\right)} \cdot \frac{\left(1-(1-z)^{2}\right)^{\frac{n+1}{2}+\kappa}}{n+1+2 \kappa} \cdot(1+\delta)^{-2}
$$


yields

$$
\begin{aligned}
J_{C}^{3} \leq\left(\begin{array}{c}
m \\
n+1
\end{array}\right) \cdot(n+1)^{\frac{3}{2}} \cdot \frac{\lambda_{n-1}\left(\omega_{n}\right)}{\lambda_{n}\left(\Omega_{n}\right)} \cdot(n+1+2 \kappa) \cdot \frac{1}{\sqrt{3}} \cdot \int_{t}^{\infty} \frac{1}{s^{2}} d s . \\
\cdot \int_{0}^{1}(1-\Phi)^{m-(n+1)} \cdot \Phi^{(n+1)-1} d \Phi \cdot(1+\delta)^{3 n+4} .
\end{aligned}
$$

Since

$$
\int_{t}^{\infty} \frac{1}{s^{2}} d s=\left[-\frac{1}{s}\right]_{t}^{\infty}=\frac{1}{t} \stackrel{t \geq 2}{\leq} \frac{1}{2}
$$

and

$$
\left(\begin{array}{c}
m \\
n+1
\end{array}\right) \cdot \int_{0}^{1}(1-\Phi)^{m-(n+1)} \cdot \Phi^{(n+1)-1} d \Phi=\frac{1}{n+1},
$$

as we already know, we finally obtain

$$
J_{C}^{3} \leq C_{3}(\kappa, n) \cdot(1+\delta)^{3 n+4}
$$

with a constant $C_{3}(\kappa, n)$. So for a given $\delta>0$ we can choose $t$ big enough und $q$ (dependent on $t$ ) small enough such that both

$$
J_{C}^{2} \leq C_{2}(\kappa, n) \cdot(1+\delta)^{n+4} \quad \text { and } \quad J_{C}^{3} \leq C_{3}(\kappa, n) \cdot(1+\delta)^{3 n+4}
$$

hold. In addition for $m$ big enough we have $J_{C}^{1} \leq(1+\delta)$. Setting

$$
C_{C}(\kappa, n):=\max \left\{1, C_{2}(\kappa, n), C_{3}(\kappa, n)\right\}
$$

we have

$$
J_{C}^{z \leq 1} \leq C_{C}(\kappa, n) \cdot\left[(1+\delta)+(1+\delta)^{n+4}+(1+\delta)^{3 n+4}\right] .
$$

As for given $\varepsilon>0$ we can choose $\delta>0$ small enough such that

$$
\left[(1+\delta)+(1+\delta)^{n+4}+(1+\delta)^{3 n+4}\right] \leq 1+\varepsilon
$$

holds, we obtain

$$
J_{C}^{z \leq 1} \leq C_{C}(\kappa, n) \cdot(1+\varepsilon)
$$

and our claim is true.

Summarizing the particular results, mainly the Theorems 2.7 and 2.17 , we can formulate our final result about the expected number of shadow vertices in the augmented rotation-symmetry-model.

Theorem 2.18. For $n \geq 3$ there is a function $\varepsilon_{\kappa}(m, n)$, which depends on the distribution parameter $\kappa$, with $\varepsilon_{\kappa}(m, n) \rightarrow 0$ for $m \rightarrow \infty, n$ constant and

$$
\mathbb{E}[\tilde{S}] \leq 2 \cdot \ln (m) \cdot(n+1)^{\frac{3}{2}} \cdot(n+3+2 \kappa)^{\frac{2}{n+1+2 \kappa}} \cdot\left(1+\varepsilon_{\kappa}(m, n)\right) .
$$




\subsection{An asymptotic lower bound}

Now, we have an upper bound for the desired expectation value. We want to work on a lower bound yet in order to get a feeling of the sharpness of our upper bound. Therefore, first we give a lower bound for the spherical measure $W$, as we cannot use the inequality of Cauchy-Schwarz in this case.

Lemma 2.19. For $z \leq 1$ there is a function $\gamma:[0,1] \rightarrow \mathbb{R}$ with

$$
W\left(b_{1}, \ldots, b_{n}\right)>\frac{1}{\sqrt{5}} \cdot \frac{1}{n ! \lambda_{n}\left(\Omega_{n}\right)} \cdot \frac{1}{z} \cdot|\operatorname{det}(B)| \cdot(1+\gamma(z))
$$

and $\gamma(z) \rightarrow 0$ for $z \rightarrow 0$.

Proof. It is clear that there is a function $\gamma:[0,1] \rightarrow \mathbb{R}$ with

$$
\lambda_{n}\left(\operatorname{conv}\left(0, b_{1}, \ldots, b_{n}\right)\right)=\lambda_{n}\left(\operatorname{conv}\left(0,\left(\begin{array}{c}
b_{1} \\
z
\end{array}\right), \ldots,\left(\begin{array}{c}
b_{n} \\
z
\end{array}\right)\right)\right) \cdot(1+\gamma(z))
$$

and $\gamma(z) \rightarrow 0$ for $z \rightarrow 0$. So we can estimate

$$
\begin{aligned}
& W\left(b_{1}, \ldots, b_{n}\right)>\frac{1}{\lambda_{n}\left(\Omega_{n}\right)} \cdot \lambda_{n}\left(\operatorname{conv}\left(0, b_{1}, \ldots, b_{n}\right)\right) \\
&= \frac{1}{\lambda_{n}\left(\Omega_{n}\right)} \cdot \lambda_{n}\left(\operatorname{conv}\left(0,\left(\begin{array}{c}
b_{1} \\
z
\end{array}\right), \ldots,\left(\begin{array}{c}
b_{n} \\
z
\end{array}\right)\right)\right) \cdot(1+\gamma(z)) \\
&=\frac{1}{\lambda_{n}\left(\Omega_{n}\right)} \cdot \frac{(n+1) !}{(n+1) !} \cdot \frac{n+1}{H\left(b_{n+1}, z\right)} \cdot \lambda_{n+1}\left(\operatorname{conv}\left(0,\left(\begin{array}{c}
b_{1} \\
z
\end{array}\right), \ldots,\left(\begin{array}{c}
b_{n+1} \\
z
\end{array}\right)\right)\right) \cdot(1+\gamma(z)) \\
&=\frac{1}{\lambda_{n}\left(\Omega_{n}\right)} \cdot \frac{1}{n !} \cdot \frac{1}{H\left(b_{n+1}, z\right)} \cdot \frac{1}{z} \cdot \operatorname{det}(B) \mid \cdot(1+\gamma(z))
\end{aligned}
$$

where $H\left(b_{n+1}, z\right)$ is the distance between $\left(b_{n+1}^{T}, z\right)^{T}$ and the hyperplane through the points $0,\left(b_{1}^{T}, z\right)^{T}, \ldots,\left(b_{n}^{T}, z\right)^{T}$. Since $\left(b_{k}^{T}, z\right)^{T}$ are all contained in a cylinder with radius 1 and height 1 , it obviously holds $H\left(b_{n+1}, z\right) \leq \sqrt{5}$ and the claim is proven.

Using Lemma 2.3 and 2.19 we obtain a lower bound for the category A.

Theorem 2.20. There is a function $\gamma:[0,1] \rightarrow \mathbb{R}$ such that for any $q \in(0,1)$ holds

$$
\begin{aligned}
J_{A}^{z \leq 1}>\left(\begin{array}{c}
m \\
n+1
\end{array}\right) \cdot(n+1) \cdot \lambda_{n-1}\left(\omega_{n}\right) \cdot \int_{q}^{1} \int_{0}^{2} G(s, z)^{m-(n+1)} \cdot \frac{s^{n}}{z^{n+1}} \cdot \Lambda(z) \cdot d s d z+ \\
+\frac{1}{\sqrt{5}} \cdot\left(\begin{array}{c}
m \\
n+1
\end{array}\right) \cdot(n+1)^{2} \cdot \frac{\lambda_{n-1}\left(\omega_{n}\right)}{\lambda_{n}\left(\Omega_{n}\right)} \cdot \int_{0}^{q} \int_{0}^{2} G(s, z)^{m-(n+1)} \cdot \frac{s^{n}}{z} \cdot \\
\cdot g_{3}(z)^{n-1} \cdot\left[g(z) g_{2}(z)-g_{4}(z)^{2}\right] \cdot(1+\gamma(z)) \cdot d s d z
\end{aligned}
$$

where $\gamma(z) \rightarrow 0$ for $z \rightarrow 0$. 
In a similar way as we obtained the upper bound we now can obtain an explicit lower bound for the category A.

Theorem 2.21. For $n \geq 3$ there is a function $\varepsilon_{\kappa}(m, n)$, which depends on the distribution parameter $\kappa$, with $\varepsilon_{\kappa}(m, n) \rightarrow 0$ for $m \rightarrow \infty, n$ constant and

$$
\begin{aligned}
J_{A}^{z \leq 1}=\left(\begin{array}{c}
m \\
n+1
\end{array}\right) \cdot(n+1) \cdot \lambda_{n-1}\left(\omega_{n}\right) \cdot \int_{0}^{1} \int_{0}^{2} G(s, z)^{m-(n+1)} \cdot \frac{s^{n}}{z^{n+1}} \cdot \Lambda(z) \cdot d s d z \\
\geq \frac{2}{\sqrt{5}} \cdot \ln (m) \cdot \frac{n(n+1)}{n+5+2 \kappa} \cdot\left(1+\varepsilon_{\kappa}(m, n)\right) .
\end{aligned}
$$

Proof. For $q \in(0,1)$ we define

$$
J_{A}^{1}:=\left(\begin{array}{c}
m \\
n+1
\end{array}\right) \cdot(n+1) \cdot \lambda_{n-1}\left(\omega_{n}\right) \cdot \int_{0}^{q} \int_{\frac{1}{m}}^{2} G(s, z)^{m-(n+1)} \cdot \frac{s^{n}}{z^{n+1}} \cdot \Lambda(z) \cdot d s d z
$$

so that obviously holds $J_{A}^{z \leq 1} \geq J_{A}^{1}$. For $J_{A}^{1}$ we have from Theorem 2.20

$$
\begin{aligned}
J_{A}^{1}>\frac{1}{\sqrt{5}} \cdot\left(\begin{array}{c}
m \\
n+1
\end{array}\right) \cdot(n+1)^{2} \cdot \frac{\lambda_{n-1}\left(\omega_{n}\right)}{\lambda_{n}\left(\Omega_{n}\right)} \cdot \int_{0}^{q} \int_{\frac{1}{m}}^{2} G(s, z)^{m-(n+1)} \cdot \frac{s^{n}}{z} \cdot \\
\cdot g_{3}(z)^{n-1} \cdot\left[g(z) g_{2}(z)-g_{4}(z)^{2}\right] \cdot(1+\gamma(z)) \cdot d s d z \\
=\frac{1}{\sqrt{5}} \cdot\left(\begin{array}{c}
m \\
n+1
\end{array}\right) \cdot(n+1)^{2} \cdot \frac{\lambda_{n-1}\left(\omega_{n}\right)}{\lambda_{n}\left(\Omega_{n}\right)} \cdot \int_{0}^{q} \int_{\frac{1}{m}}^{2} G(s, z)^{m-(n+1)} \cdot s^{n} . \\
\quad \cdot \frac{g(z)-g_{4}(z)}{z} \cdot g_{3}(z)^{n-1} \cdot \frac{\left[g(z) g_{2}(z)-g_{4}(z)^{2}\right]}{g(z)-g_{4}(z)} \cdot(1+\gamma(z)) \cdot d s d z .
\end{aligned}
$$

From [8] and Lemma 2.6 we know that for any $\delta>0$ we can choose $q>0$ depending on the dimension $n$ and the distribution parameter $\kappa$ small enough such that for any $z \in[0, q]$ holds

1. $g_{3}(z) \geq \frac{\Phi(s, z)}{s} \cdot(1-\delta)$,

2. $1+\gamma(z) \geq 1-\delta$,

3. $g(z) g_{2}(z)-g_{4}(z)^{2} \geq\left[\frac{\Phi(s, z)}{s}\right]^{2} \cdot \frac{n+1+2 \kappa}{n+5+2 \kappa} \cdot(1-\delta)$

4. $g(z)-g_{4}(z) \leq \frac{\Phi(s, z)}{s} \cdot\left(\frac{n+1}{2}+\kappa\right) \cdot(1-\delta)^{-1}$. 
So for a suitable $q$ we obtain

$$
\begin{aligned}
& J_{A}^{1}>\frac{2}{\sqrt{5}} \cdot\left(\begin{array}{c}
m \\
n+1
\end{array}\right) \cdot \frac{(n+1)^{2}}{n+5+2 \kappa} \cdot \frac{\lambda_{n-1}\left(\omega_{n}\right)}{\lambda_{n}\left(\Omega_{n}\right)} \\
& \cdot \int_{\frac{1}{m}}^{2} \int_{0}^{q}[1-\Phi(s, z)]^{m-(n+1)} \cdot \Phi(s, z)^{n-1} \cdot \frac{\Phi(s, z)}{s} \cdot \frac{\left[g(z)-g_{4}(z)\right] s}{z} \cdot d z d s \cdot(1-\delta)^{n+2} \\
&=\frac{2}{\sqrt{5}} \cdot\left(\begin{array}{c}
m \\
n+1
\end{array}\right) \cdot \frac{(n+1)^{2}}{n+5+2 \kappa} \cdot \frac{\lambda_{n-1}\left(\omega_{n}\right)}{\lambda_{n}\left(\Omega_{n}\right)} \cdot \\
& \cdot \int_{\frac{1}{m}}^{2} \int_{0}^{\Phi(s, q)}(1-\Phi)^{m-(n+1)} \cdot \Phi^{(n+1)-1} \cdot \frac{1}{s} \cdot d \Phi d s \cdot(1-\delta)^{n+2} .
\end{aligned}
$$

Since

$$
\begin{aligned}
\left(\begin{array}{c}
m \\
n+1
\end{array}\right) \cdot \int_{\Phi(s, q)}^{1}(1-\Phi)^{m-(n+1)} \cdot \Phi^{(n+1)-1} d \Phi & \\
& \leq m^{n+1} \cdot(1-\Phi(s, q))^{m-(n+1)} \int_{0}^{\Phi(s, q)} \Phi^{(n+1)-1} d \Phi \stackrel{m \rightarrow \infty}{\longrightarrow} 0,
\end{aligned}
$$

we have for given $\varepsilon>0, q$ small enough and $m$ big enough

$$
\begin{aligned}
J_{A}^{1} \geq \frac{2}{\sqrt{5}} \cdot\left(\begin{array}{c}
m \\
n+1
\end{array}\right) \cdot \frac{(n+1)^{2}}{n+5+2 \kappa} \cdot \frac{\lambda_{n-1}\left(\omega_{n}\right)}{\lambda_{n}\left(\Omega_{n}\right)} . \\
\cdot \int_{0}^{1}(1-\Phi)^{m-(n+1)} \cdot \Phi^{(n+1)-1} d \Phi \cdot \int_{\frac{1}{m}}^{2} \frac{1}{s} d s \cdot(1-\varepsilon) .
\end{aligned}
$$

Now, we use the known equations, which can be read in the appendix of [4],

$$
\begin{aligned}
& \text { 1. } \frac{\lambda_{n-1}\left(\omega_{n}\right)}{\lambda_{n}\left(\Omega_{n}\right)}=n, \\
& \text { 2. }\left(\begin{array}{c}
m \\
n+1
\end{array}\right) \cdot \int_{0}^{1}(1-\Phi)^{m-(n+1)} \cdot \Phi^{(n+1)-1} d \Phi=\frac{1}{n+1}
\end{aligned}
$$

as well as the already known calculation

$$
\int_{\frac{1}{m}}^{2} \frac{1}{s} d s=[\ln (s)]_{\frac{1}{m}}^{2}=\ln (m)+\ln (2) \geq \ln (m) .
$$


So finally we obtain

$$
J_{A}^{1} \geq \frac{2}{\sqrt{5}} \cdot \ln (m) \cdot \frac{n(n+1)}{n+5+2 \kappa} \cdot\left(1+\varepsilon_{\kappa}(m, n)\right)
$$

and the claim is obvious.

Using this result we can formulate our lower bound for the whole expectation value.

Theorem 2.22. For $n \geq 3$ there is a function $\varepsilon_{\kappa}(m, n)$, which depends on the distribution parameter $\kappa$, with $\varepsilon_{\kappa}(m, n) \rightarrow 0$ for $m \rightarrow \infty, n$ constant and

$$
\mathbb{E}[\tilde{S}] \geq \frac{2}{\sqrt{5}} \cdot \ln (m) \cdot \frac{n(n+1)}{n+5+2 \kappa} \cdot\left(1+\varepsilon_{\kappa}(m, n)\right) .
$$

Comparing the asymptotic bounds we see that our upper bound has the form $\ln (m) \cdot n^{\frac{3}{2}}$, while the lower bound has the form $\ln (m) \cdot n$. So we only have a gap in the constant factor of size $\sqrt{n}$, but we can state that the order of growth of $m$ is sharp.

\section{Conclusion}

As we successfully have performed the analysis of the expected number of shadow vertices in the augmented rotation-symmetry-model, the main task of this paper, we want to give the result for the augmented dimension-by-dimension-algorithm and briefly discuss our issues.

\subsection{The result for the whole algorithm}

In the previous section we have analyzed the expected number of shadow vertices in the augmented rotation-symmetry-model and have obtained an asymptotic upper bound for the last stage of the augmented dimension-by-dimension-algorithm of the form $\ln (m) \cdot n^{\frac{3}{2}}$. For the first $n$ stages, for which we have entire rotation symmetry, from Höfner [9] the asymptotic upper bound

$$
C_{O} \cdot m^{\frac{1}{n+1+2 \kappa}} \cdot n^{2} \cdot(n+1+2 \kappa)^{\frac{1}{2}}
$$


is known where $C_{O}$ is a constant not depending on $m, n$ or $\kappa$. Since our result for the last stage offers a smaller asymptotic size of growth in $m$, we obtain the following result for the complete algorithm.

Theorem 3.1. For $n \geq 3$ there is a function $\varepsilon_{\kappa}(m, n)$, which depends on the distribution parameter $\kappa$, with $\varepsilon_{\kappa}(m, n) \rightarrow 0$ for $m \rightarrow \infty$, $n$ constant and

$$
\begin{aligned}
& \mathbb{E}_{m, n}[\text { "Number of steps of the augmented algorithm" }]=\mathbb{E}_{m, n}\left[s_{t}\right] \\
& \quad \leq C \cdot m^{\frac{1}{n+1+2 \kappa}} \cdot n^{2} \cdot(n+1+2 \kappa)^{\frac{1}{2}} \cdot\left(1+\varepsilon_{\kappa}(m, n)\right)
\end{aligned}
$$

where $C$ is a constant not depending on $m, n$ or $\kappa$.

This upper bound is sharp in the quantities $m, n$ and $\kappa$, as we have by Höfner [9] the asymptotic lower bound for the first $n$ stages

$$
C_{U} \cdot m^{\frac{1}{n+1+2 \kappa}} \cdot n^{2} \cdot(n+1+2 \kappa)^{\frac{1}{2}}
$$

where $C_{U}$ is a constant not depending on $m, n$ or $\kappa$.

\subsection{Summary}

As we have seen, the additional stage of our augmented dimension-by-dimensionalgorithm, which was necessary to consider also problems with negative capacities (right hand sides), is harmless compared to the whole effort of the previous stages solving the problem in the asymptotic case, when $n$ is constant and $m$ tends to infinity. It would be very interesting whether this result still holds for the moderate case, when $n$ and $m$ are constant or of comparable size. Although this seems to be very obvious, a rigorous mathematical proof seems to be extremely hard. The reason is that the methods used in the rotational symmetric case cannot be directly transfered in that moderate case, especially not to those positions of the hyperplane that lie nearly parallel to the cylinder cover. So there must be found new methods for that case. Rather interesting are our single asymptotic results for the different categories of hyperplanes. For the category A we have an upper bound in the form $\ln (m) \cdot n^{\frac{3}{2}}$, while for the categories B and C we have constant (in $m$ ) bounds. Since we consider the projection of the polyhedron onto a two-dimensional shadow plane, it suggests itself to compare our results with some basic results for the two-dimensional case from Renyi and Sulanke [11]. Doing so, we see that both results agree in that sense that the cases in which one "vertex" is cut (category A) are the relevant ones for the asymptotic order of growth $\ln (m)$, while the other cases only account for a constant (in $m$ ). Keep in mind that category B and C are identical, if there are only two dimensions. So our results seem to be plausible, even if they are based on a complete different stochastical model, of course. 


\section{References}

[1] K. H. Borgwardt: A Sharp Upper Bound for the Expected Number of ShadowVertices in the Rotation-Symmetry-Model - Quadratic in the Smaller and Sublinear in the Larger Dimension of the LP, Mathematics of Operations Research 24/4: 925-984, 1999.

[2] K. H. Borgwardt: Average-Case Analysis of the Double Description Method and the Beneath-Beyond Algorithm, Discrete \& Computational Geometry 37: 175-204, 2007.

[3] K. H. Borgwardt: The Average Number of Pivot Steps Required by the SimplexMethod is Polynomial, Zeitschrift für Operations Research 26: 157-177, 1982.

[4] K. H. Borgwardt: The Simplex Method: A Probabilistic Analysis, Springer, Berlin, 1987.

[5] K. H. Borgwardt: Untersuchungen zur Asymptotik der mittleren Schrittzahl von Simplexverfahren in der linearen Optimierung, Dissertation, Universität Kaiserslautern, 1977.

[6] G. B. Dantzig: Linear Programming and Extensions, Princeton University Press, Princeton, 1963.

[7] G. B. Dantzig: Maximization of a Linear Function of Variables subject to Linear Inequalities, in Activity Analysis of Production and Allocation, Cowles Commission Monograph 13: 339-347, John Wiley \& Sons Inc., New York, 1951.

[8] M. Göhl: Der durchschnittliche Rechenaufwand des Simplexverfahrens unter einem verallgemeinerten Rotationssymmetriemodell, Dissertation, Logos, Berlin, 2013.

[9] G. Höfner: Lineare Optimierung mit dem Schatteneckenalgorithmus: Untersuchungen zum mittleren Rechenaufwand und Entartungsverhalten, Dissertation, Wißner, Augsburg, 1995.

[10] V. Klee, G. J. Minty: How Good is the Simplex Algorithm?, Inequalities III: 159-175, Academic Press, 1972.

[11] A. Renyi, R. Sulanke: Über die konvexe Hülle von $n$ zufällig gewählten Punkten, Zeitschrift für Wahrscheinlichkeitstheorie 2: 75-84, 1963. 